\title{
Preparación de la muestra: un paso crucial para el análisis por GC-MS
}

\author{
Elena E. Stashenko*, Jairo René Martínez \\ Laboratorio de Cromatografía, Centro de Investigación de Excelencia, CENIVAM, \\ Escuela de Química, Universidad Industrial de Santander, UIS, Bucaramanga, Colombia \\ e-mail:elena@tucan.uis.edu.co
}

\section{Resumo}

Se destaca la importancia de la preparación de la muestra en los análisis GC y GC-MS. Se ilustran con ejemplos las consecuencias de algunos errores en la preparación de la muestra, sobre los perfiles cromatográficos de los extractos resultantes. Se describen las etapas principales de la cadena de eventos que conducen a un extracto que permita determinar cromatográficamente los analitos de interés en una muestra. Se incluye una breve revisión de los métodos de extracción más comunes, sus alcances y campos de aplicación. El uso de blancos positivos y negativos, sustancias sucedáneas de los analitos y otras recomendaciones para la prevención de la contaminación cruzada, la mejora de la recuperación y la obtención de extractos con pocas interferencias, se presentan junto con explicaciones sobre su conveniencia y efectos en el resultado final del análisis GC-MS.

Palavras-chave

GC; GC-MS; preparación de la muestra; técnicas de extracción.

\section{Sample preparation: a crucial step in GC-MS analysis}

\section{Abstract}

The importance of sample preparation in GC and GC-MS analyses is highlighted. Examples of chromatographic profiles are used to illustrate the consequences of certain sample preparation mistakes. The main steps of the chain of events that leads to a suitable extract are described, in the context of the chromatographic determination of target analytes. A brief revision is made of the most common extraction methods, their scope and application fields. The use of positive and negative blanks, as well as surrogate substances and other recommendations for recovery improvement, the prevention of cross contamination, and the achievement of extracts containing few interferences, are presented together with explanations about their convenience and effects on the final analytical results.

Keywords

GC; GC-MS; sample preparation; extraction techniques. 
Profesor: El cromatograma obtenido por GC-MS no se ve bien, ya que presenta muchos picos deformados, asimétricos, con deriva de la línea base y un ruido excesivo; además en los espectros de masas se registran señales intrusas, que no son propias de las sustancias-target presentes en el extracto.

Estudiante: Sí, profesor, efectivamente, son la columna y el equipo que usé; ambos fallan; toca llamar al técnico, para que venga urgentemente y los revise.

Profesor: $\quad$ No, señor, no creo que la causa sea el equipo. La columna se ha instalado recientemente, es nueva; el septum y el liner también se han cambiado hace poco, la cámara de ionización se limpió un par de días atrás y el electromultiplicador es nuevo. El problema serán sus manos.

Estudiante: ¡Mis manos, profesor! ¿Qué quiere decir con ello?

Profesor: Sencillamente, la preparación de la muestra falló, es decir, que fue deficiente y poco esmerada; hay muchas interferencias y contaminantes presentes en los extractos que inyectó, se encuentran en éstos sustancias que no son compatibles con el sistema GC-MS. Hay que repetir la extracción, hacer limpieza del extracto y concentrarlo. Primero, debemos buscar la falla en nosotros mismos y luego, compañero, echemos la culpa al equipo analítico. Como se dice en un refrán "Garbage in, garbage out". A trabajar entonces, manos a la obra.

\section{Introducción}

La finalidad de muchas propuestas de investigación es buscar fondos para adquirir un equipo analítico nuevo y de alta tecnología. Entre las máquinas "obligatorias" en un laboratorio instrumental siempre figura un cromatógrafo de gases (GC) acoplado a un espectrómetro de masas (MS), GC-MS. En este instrumento, se unen dos poderosas técnicas, la de separación (GC) y la espectroscópica (espectrometría de masas, MS), que se usan no sólo para detectar y cuantificar los analitos de un extracto multicomponente sino para identificarlos ${ }^{1-4}$. La técnica GC-MS, a pesar de sus grandes avances y desarrollos, multifuncionalidad, sensibilidad y reproducibilidad alta, así como la facilidad de manejo y robustez, posee una limitación inherente, que reduce el número de sustancias que puede analizar, porque no todos los compuestos pueden ingresar al sistema GC-MS, sin que se empeore su funcionamiento o la detección sensible. Esta limitación está rela- cionada con la volatilidad, la termoestabilidad, la polaridad y el peso molecular alto de los analitos de interés. En otras palabras, para su análisis por GC-MS, los analitos-target deben ser estables hasta temperaturas de $300-350{ }^{\circ} \mathrm{C}$, volátiles o volatilizables, i.e., existir en la fase gaseosa, sin experimentar ninguna modificación química, $y$, por lo general, su peso molecular no debe superar 500-550 Da. Típicamente, por GC-MS se analizan moléculas no polares y medianamente polares con masas moleculares de hasta 400-450 Da. Todo ello implica que menos del $10 \%$ de todas las moléculas existentes pueden ser analizadas por GC-MS. En términos del número total de sustancias existentes en el planeta o sintetizadas en el laboratorio -y que son aptas para el análisis por GC-MS-, esta cifra es alta, pero si se refiere a la fracción porcentual que representan de todas las especies moleculares existentes, este valor es relativamente bajo. Por ende, la operación de un equipo GC-MS en el laboratorio debe ser acompañada de una infraestructura de pre- 
paración de la muestra necesaria para garantizar el ingreso al aparato de sólo aquellas sustancias que son estrictamente compatibles con las técnicas GC o GC-MS. Suena obvio y trivial, pero, en la práctica, este hecho a menudo se menosprecia y ello crea problemas analíticos, ya que puede empeorar los resultados obtenidos por la técnica GC-MS, generando a menudo los llamados falsos negativos (no se detectan los analitos presentes, no hubo previa concentración y limpieza del extracto) o falsos positivos (se presenta una confusión con las señales de interferencias que se toman por las del analito).

Las limitaciones en cuanto a la naturaleza química de las sustancias "compatibles" con la técnica GC-MS, involucran la necesidad de su aislamiento previo (extracción) a partir de la muestra (matriz) real. En la mezcla multicomponente, se encuentran generalmente tanto los analitos "amenos" al sistema instrumental GC-MS, como los no aptos para este análisis. A menudo, los componentes de interés se encuentran en concentraciones a nivel de trazas y se necesita un procedimiento de enriquecimiento (concentración y limpieza) del extracto, para poder detectarlos. El tipo y la diversidad de mezclas complejas -que se procesan-, es muy amplio e incluye muestras ambientales (aire, aguas, suelos), de origen biológico (especies vegetales, tejidos, células, fluidos fisiológicos, etc.), alimentos y bebidas, productos de química combinatoria, artículos de uso personal, drogas y evidencias forenses (residuos de incendios, explosivos, pinturas, etc.), entre muchos otros.

Entre los compuestos analizables por GC-MS figuran los de interés ambiental ${ }^{5,6}$, aromas y fragancias ${ }^{7,8}$, drogas y sus metabolitos ${ }^{9-11}$, pesticidas $^{12}$, solventes, acelerantes de incendios maliciosos $^{13}$, explosivos ${ }^{14,15}$, aditivos de bajo y mediano peso molecular presentes en distintos productos finales, e.g., en polímeros ${ }^{16} \mathrm{o}$ alimen- $\operatorname{tos}^{17,18}$, semioquímicos (feromonas) ${ }^{19}$ y productos naturales ${ }^{20,21}$, entre otros. El sistema GC-MS puede ser acoplado a diferentes dispositivos adicionales, e.g., muestrador de espacio de cabeza (Headspace Sampler, HS) ${ }^{22,23}$, equipo de purga y trampa (P\&T), un pirolizador, un horno para desorción térmica (Thermal desorption, TD), etc.

El mundo que nos rodea -y cuyas pequeñas "fracciones" o procesos son objetos de numerosos estudios analíticos-, es una mezcla compleja de miles a millones de sustancias de naturaleza química muy diversa. Por ejemplo, tan sólo el aroma de café puede contener más de 800 sustancias químicas diferentes, prácticamente, de todas las clases de compuestos orgánicos y con diversas funcionalidades, e.g., hidrocarburos, alcoholes, aldehídos, ácidos, éteres, ésteres, heterociclos, etc. $^{24}$. Las fragancias florales, extractos frutales y aceites esenciales pueden estar compuestos por centenares de sustancias ${ }^{25,26}$; el petróleo constituye otro ejemplo de una mezcla complejísima, que posee $10^{5}-10^{6}$ sustancias, desde un simple metano hasta agregados macromoleculares de alto peso molecular (asfaltenos, resinas) de muy complicada caracterización química ${ }^{27}$. Hay más de 100 mil proteínas en el cuerpo humano. Entre diversos productos naturales, se han caracterizado miles de terpenos y terpenoides ${ }^{28,29}$, derivados fenólicos y alcaloides ${ }^{30}$. Los ejemplos se pueden continuar, pero la idea principal es clara: en un laboratorio analítico se manejan -casi sin excepción- mezclas, a menudo muy complejas, aunque en su interior, de interés analítico, pueden figurar sólo alguno(s) compuesto(s) o analitostarget (e.g., plaguicidas, fármacos o sus metabolitos, sustancias responsables por un off-flavour, etc.) o sus homólogos y derivados. Típicamente, se requiere determinar su presencia e identificarlos (análisis cualitativo), pero frecuentemente también hallar su respectiva cantidad (análisis cuantitativo). El análisis cualitativo se lleva a 
cabo por GC-MS operado en modo de barrido completo (full scan), mientras que la cuantificación del analito se hace, por lo general, en modo de monitoreo de ion(es) seleccionado(s) (SIM, Selected Ion Monitoring, por sus siglas en inglés) o, en el caso de la configuración MS tándem, por ejemplo, con analizadores de triple cuadrupolo, QqQ, a través del monitoreo de reacción múltiple (MRM, Multiple Reaction Monitoring, por sus siglas en inglés).

No existe una técnica analítica instrumental completamente universal (es decir, que sirva para todas las matrices) para separar, detectar, cuantificar o identificar inequívocamente todos los analitos-target presentes en una mezcla compleja. Tampoco existe una técnica instrumental capaz de hacerlo directamente, sin tratamiento previo (aunque mínimo) de la muestra, i.e., sin llevar a cabo los procesos de extracción, concentración, fraccionamiento o la limpieza del extracto. Por ejemplo, antes de someterlos al análisis por GC-MS del aroma de una carne frita, los compuestos volátiles deben ser aislados de la matriz, ya que a la columna cromatográfica capilar no deben ingresar proteínas, triglicéridos o ácidos grasos. Cuando se necesita, por ejemplo, determinar los residuos de drogas o sus metabolitos en una muestra de orina, una alícuota de ésta no se introduce directamente al equipo GC o GC-MS, porque ni agua, ni sales presentes en la orina son compatibles con este instrumento y deben ser excluidas del extracto, antes de su inyección. El agua, las sales orgánicas e inorgánicas, las sustancias termolábiles o de alto peso molecular (e.g., proteínas, sacáridos, glicéridos, glucósidos, ceras, etc.), compuestos muy polares (azúcares, polifenoles, aminoácidos, etc.) no son aptos para el análisis por GC-MS y, si eventualmente resultan ingresando al equipo, afectan su resolución, sensibilidad e imposibilitan la identificación de analitos; la calidad del análisis puede empeorar irreversiblemente, mientras que el funcionamiento del mismo equipo se compromete severamente (y no es la "culpa" del equipo, como en muchos casos se suele suponer $)^{31}$.

Lo expuesto anteriormente conduce a la conclusión de que la muestra -para su análisis por GC-MS-, inevitablemente debe "adecuarse". Este proceso se llama "preparación de la muestra" (Sample Preparation) y conforma uno de los pasos más importantes en toda cadena analítica. La etapa de preparación de la muestra en la cadena analítica puede ocupar del 60 al 85\% del tiempo total de análisis. Del éxito de esta etapa dependerán parámetros analíticos tan importantes como la eficiencia de recuperación de analitos, la reproducibilidad y la robustez de la técnica, así como la sensibilidad y selectividad de todo el método, el tiempo requerido para el análisis y su costo, etc. La robustez de la cadena analítica depende, por lo general, de la fortaleza de su eslabón más débil, que en muchos casos, precisamente lo conforma la fase de preparación de la muestra, i.e., la obtención de un extracto enriquecido con analito(s)-target e "idealmente" libre de otros componentes de la matriz o interferencias incompatibles con el equipo GC-MS.

En la gran mayoría de currículos académicos universitarios en el mundo, infortunadamente, si más bien se dictan diferentes cursos de Análisis Instrumental (Cromatografía, Espectroscopia, Espectrometría de Masas, Resonancia Magnética Nuclear, Difracción de Rayos X, etc., Análisis Forense, Ambiental, de Alimentos, etc.) y de las estrategias de Identificación de Compuestos Orgánicos, la asignatura Preparación de Muestras, rara vez aparece como obligatoria. No obstante, la calidad de un análisis instrumental, el funcionamiento y el tiempo de vida útil de un equipo analítico (e.g., GC-MS), la frecuencia de su mantenimiento preventivo y correctivo son parámetros derivados directamente de la "calidad" de la 
muestra (extracto) introducida, verbigracia, de su cantidad, grado de limpieza y concentración de analitos, es decir, de su "adecuación" previa al análisis. En este caso, la pirámide de errores o posibles fallas en la cadena del análisis instrumental tendrá en su base la competencia (knowhow) del operador y la preparación adecuada de la muestra (Figura 1). La mayoría de las fallas que se presentan en el funcionamiento del equipo analítico (descartando, por supuesto, aquellas "genéticas" provenientes de su fabricación) se derivan típicamente de: 1) su operación correcta, i.e., mantenimiento preventivo, cumplimiento de recomendaciones para su uso, estabilidad de la corriente eléctrica, etc., $y$, fundamentalmente 2) el tipo de la muestra (preparación, concentración y limpieza adecuada del extracto), que se introduce al sistema GC-MS.

Cualquier muestra a procesar se puede concebir como un sistema dinámico (Figura 2), en donde interactúan tres "esferas" correspondientes a sus "bloques" principales, a saber: 1) la matriz, 2) las interferencias y 3) los analitos-tar- get. El aislamiento cuantitativo de los últimos es el objetivo principal de la preparación de la muestra. La meta es separar los analitos cuantitativamente, sin alterarlos o modificarlos químicamente, de manera predecible y reproducible, dejando el extracto final libre de los componentes de la matriz y de las interferencias. La cantidad de analitos separados con respecto a su cantidad original en la muestra refleja la eficiencia de su extracción. El aislamiento cuantitativo de los analitos-target se hace más fácil y eficiente cuando sus propiedades físico-químicas (e.g. temperatura de ebullición, polaridad, etc.) son muy diferentes de las de otros componentes de la matriz; por ejemplo, es más fácil aislar un hidrocarburo del agua que un fenol presente en ésta.

A pesar de que frecuentemente se logran separar, con alta eficiencia, los analitos de la matriz donde se encuentran (e.g., aire, agua, suelo, alimento, fluido biológico, tejido, etc.), en el extracto final, empero, pueden "sobrevivir" las interferencias, verbigracia, aquellas sustancias "oportunistas" que poseen una afinidad dual,

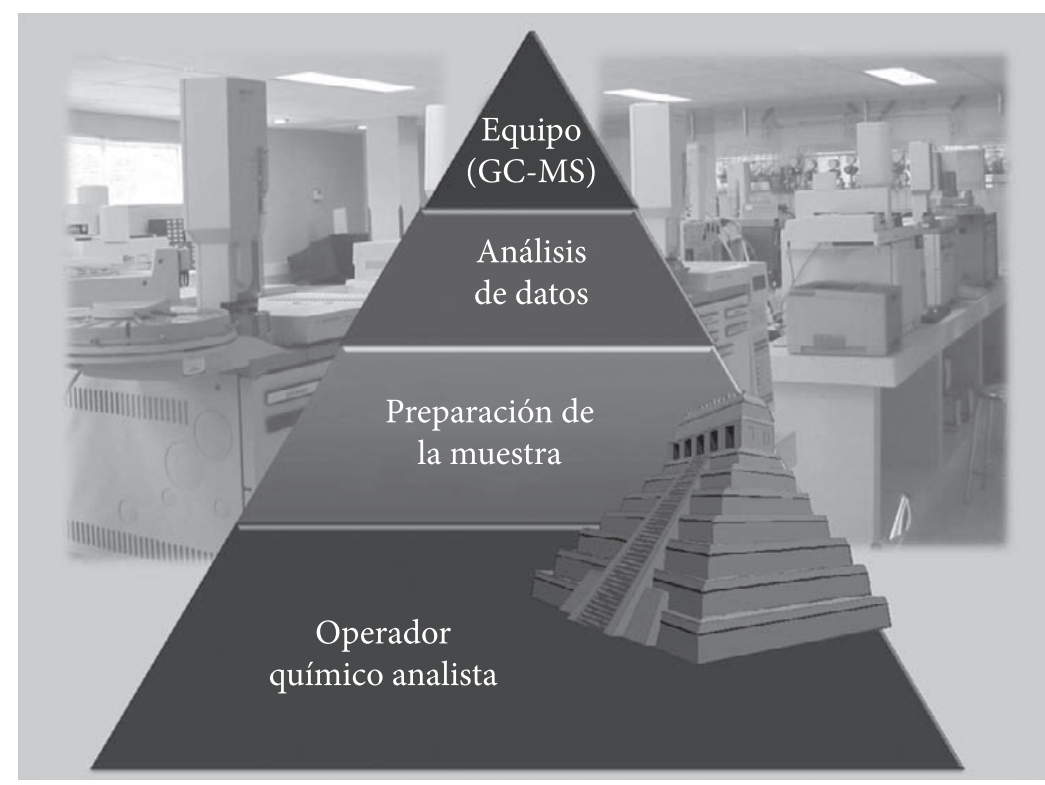

Figura 1 Las causas y la magnitud de los posibles errores o fallas que puedan surgir en una cadena analítica, representado alegóricamente como una pirámide, que culmina con el respectivo análisis instrumental (e.g., GC-MS) del extracto obtenido. 
tanto por la matriz (e.g., agua, grasa), como por el medio extractivo (e.g., disolvente, adsorbente). En este caso, el análisis por GC-MS puede afectarse por su presencia, porque las interferencias pueden disminuir la resolución, la selectividad

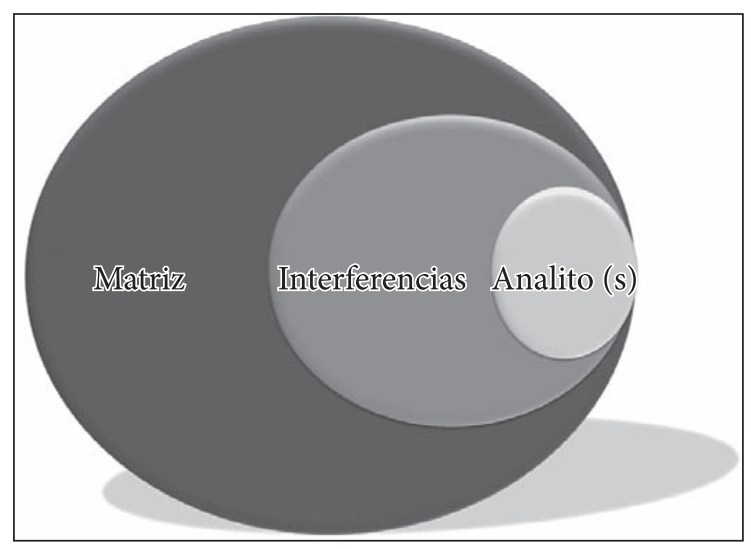

Figura 2 La "estructura" típica de una muestra: matriz, analitos-target e interferencias. Las interferencias durante el proceso de extracción de analitos-target revelan su naturaleza dual, ya que pueden tener afinidad tanto por la matriz, como por el medio extractivo (e.g., por un disolvente, un adsorbente, gas de purga, etc.). $y$, fundamentalmente, la sensibilidad del equipo GC-MS, ya que generan la contaminación progresiva del sistema analítico, dejan en él la "memoria" (efecto carry-over) o simplemente se superponen a las señales generadas por el analito-target, imposibilitando su identificación o cuantificación.

En la Figura 3 se reflejan, de una manera muy simplificada, los posibles resultados de la extracción de una muestra compleja (Figura 2), representados así: a) La separación de analitos es deficiente, con bajo porcentaje de recuperación y sin la debida limpieza (clean-up) del extracto (es decir, sin eliminación de otros componentes de la matriz e interferencias que afectarán negativamente el análisis por GC-MS); b) La eficiencia de extracción es mucho más alta, aunque se requiere una limpieza adicional del extracto, ya que aún perduran en éste las interferencias; c) La extracción es casi perfecta: se observa una alta eficiencia (porcentaje de recuperación de analitos) y muy buena limpieza del extracto. Si el

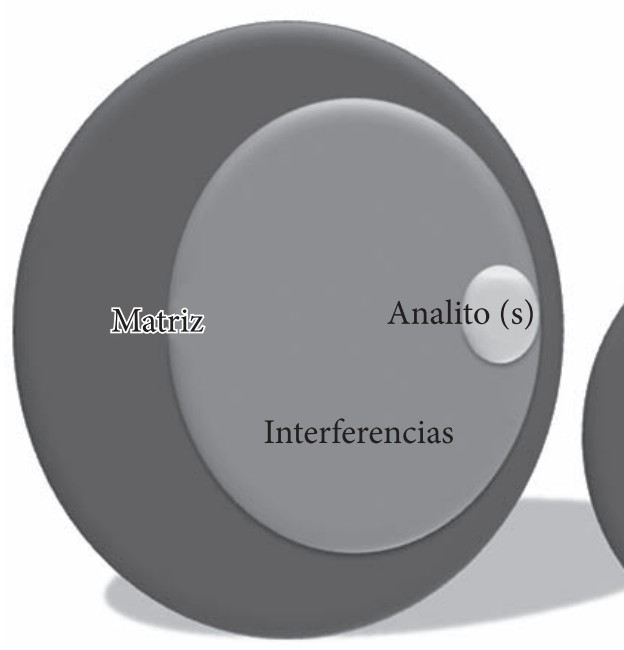

(a)

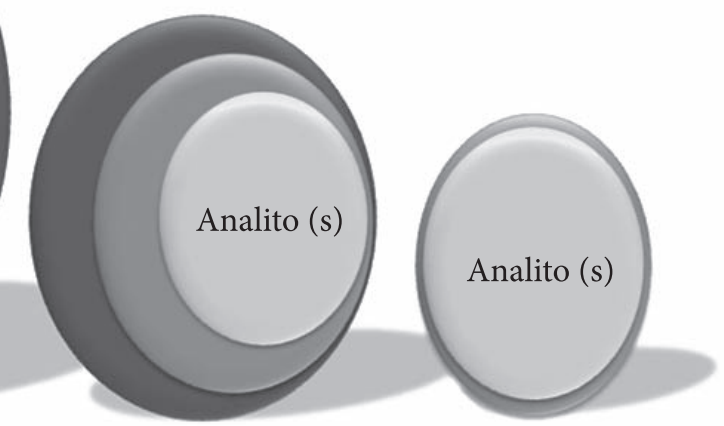

(b) (c)

Figura 3 Representación esquemática de los resultados posibles de una técnica de extracción. a) Extracción deficiente con recuperación de analitos-target baja y limpieza del extracto deficiente. b) Extracto caracterizado por una buena recuperación de analitos-target, pero requiere una mejor limpieza (aislamiento de componentes de la matriz e interferencias). c) Extracción adecuada, con una eficiencia de recuperación de analitos alta y una limpieza del extracto muy buena. 
extracto obtenido (Figura 3c) está conformado por analitos de bajo peso molecular $(<400 \mathrm{Da})$, con volatilidad alta o mediana, termoestables y no muy polares, éste es el extracto perfecto, apto para el análisis por GC-MS. La siguiente tarea en la cadena analítica consistirá en la escogencia correcta de la columna (fase estacionaria, dimensiones) y de los parámetros operacionales del equipo GC-MS (modo de inyección, temperaturas, flujos de gases, rango de masas, velocidad de barrido, modo de adquisición de la corriente iónica, full scan o SIM, etc.) ${ }^{32,33}$. "Idealmente", la técnica de extracción debe ser exhaustiva y altamente selectiva, y permitir el aislamiento únicamente de analitos de interés; sin embargo, ello no siempre es posible. A menudo, hay que recurrir también a un procedimiento adicional, i.e. a la derivación química de analitos, verbigracia, su modificación química ${ }^{34,35}$; por ejemplo, la metilación de ácidos grasos, acilación de aminas, sililación de azúcares; todo ello, con el fin de incrementar la selectividad y la sensibilidad de su detección, aumentar su estabilidad térmica y volatilidad (de los derivados), y mejorar sus características cromatográficas (tiempos de retención más cortos, forma de pico, su simetría, etc.). Debido a la importancia que tiene la etapa de preparación correcta de la muestra, varios libros especiales y excelentes monografías $^{36-39}$, revisiones temáticas ${ }^{40-45}$ se han dedicado a este tema. En éstos, se analizan aspectos más relevantes de varias técnicas de preparación de la muestra, incluyendo métodos modernos, para el análisis de extractos o fracciones volátiles por GC-MS.

\section{Observaciones generales}

La cadena analítica típicamente contiene los siguientes cinco pasos: 1) Muestreo (Sampling); 2) Preparación de la muestra (Sample Preparation), que incluye pre-tratamiento, extracción, concentración y limpieza (clean-up); 3) Separación (Separation), por regla general, cromatográfica; 4) Detección (Detection), que puede incluir métodos ópticos, espectrométricos, electroquímicos, radioquímicos, inmunoensayos, etc.; 5) Análisis de datos (Data Analysis) y su interpretación; validación estadística de resultados que conduce luego a la toma de decisión sobre los posibles cambios en la metodología utilizada, con la subsiguiente repetición o no del procedimiento realizado. En todos los casos, es menester conservar (adecuadamente) la contra-muestra (duplicado) para las posibles repeticiones del ensayo o controversias que pueden presentar los resultados obtenidos.

Las tendencias modernas en el desarrollo de la cadena analítica se basan en los conceptos de: a) Miniaturización; b) Integración de varios procesos y su Automatización (extracción, limpieza, concentración, análisis); c) Hyphenation (acoplamiento de técnicas de preparación con diferentes métodos de separación y detección, por ejemplo, HS-SPME-GC/MS, P\&T-GC/MS o SPE-LC/MS, GCxGC-MS-TOF, LC-GC, entre muchos otros) e (d) Implementación de métodos de extracción basados en la filosofía de preparación de la muestra QuEChERS ("catchers") y de química verde. Muchos métodos de preparación de la muestra, hoy en día, sobre todo, para el análisis de residuos de pesticidas en diferentes matrices, se diseñan acorde con la filosofía QuEChERS ${ }^{46-49}$ (Figura 4); es cuando se minimiza el uso de solventes y el análisis instrumental se hace con detectores selectivos (de captura de electrones, ECD; selectivo de nitrógeno y fósforo, NPD; fotométrico de llama, FPD; de quimioluminiscencia, entre otros) o por GC-MS operado en modo SIM o en modo MRM, cuando se usan las configuraciones tándem, por ejemplo, analizador de triple cuadrupolo, QqQ. Otra tendencia moderna importante es implementar las técnicas solventless (sin uso de solven- 


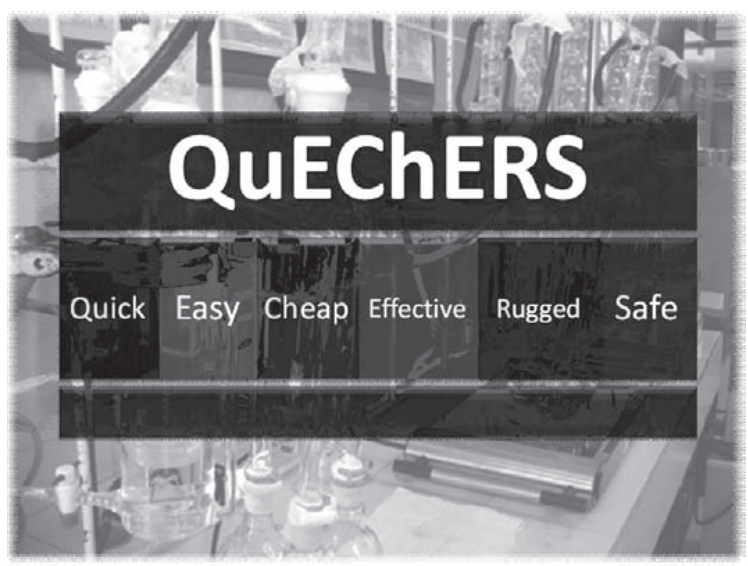

Figura 4 Muchos métodos de preparación de la muestra, hoy en día, sobre todo para el análisis de residuos de pesticidas, están diseñados de acuerdo con la filosofía de técnicas QuEChERS, que abarcan los métodos de extracción rápidos (Quick), fáciles de llevar a cabo (Easy), económicos (Cheap), efectivos (Effective), robustos (Rugged) y, sobre todo, seguros (Safe).

tes), basadas en el monitoreo de analitos en fase vapor directamente o usando crio-atrapamiento, sorbentes sólidos o membranas; en general, el enfoque es buscar y optimizar los métodos de extracción rápidos, efectivos, robustos y seguros.

En la mayoría de los casos, la extracción de analitos se acompaña de su concentración en un extracto final (rotoevaporación, flujo de gas inerte, evaporadores especiales, equipos tipo Kuderna-Danish, etc.) (Figura 5) y de la limpieza (clean-up) que permita aislar las interferencias (cromatografía en columna, extracción en fase sólida, SPE; cromatografía líquida, LC; cromatografía de permeación en gel, GPC; etc.). La etapa de clean-up es necesaria para evitar que las interferencias (proteínas, grasa, azufre, sales, resinas, otros) ingresen a los equipos GC o GC-MS. Se busca siempre en la preparación de la muestra la técnica que más se acerca a la "ideal”, verbigracia, que sea altamente selectiva y sensible, eficiente y fácil de llevar a cabo, además, que debe ser rápida, robusta y económica y con la posibilidad

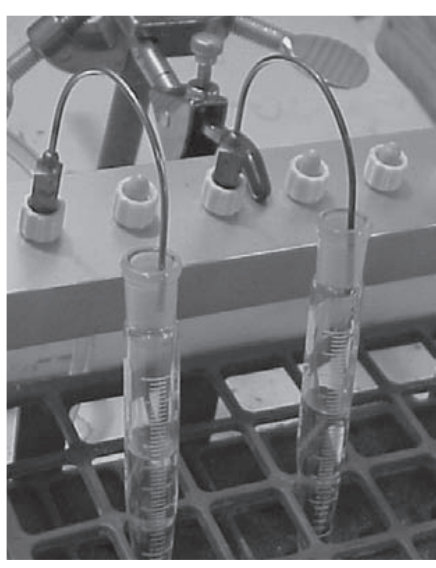

(a)

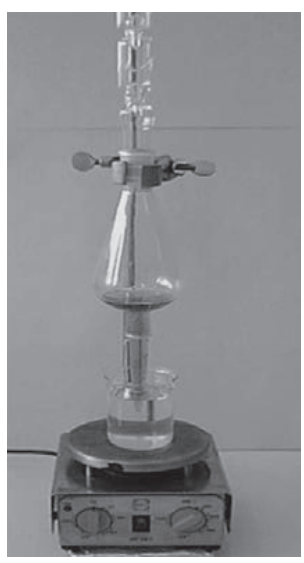

(b)

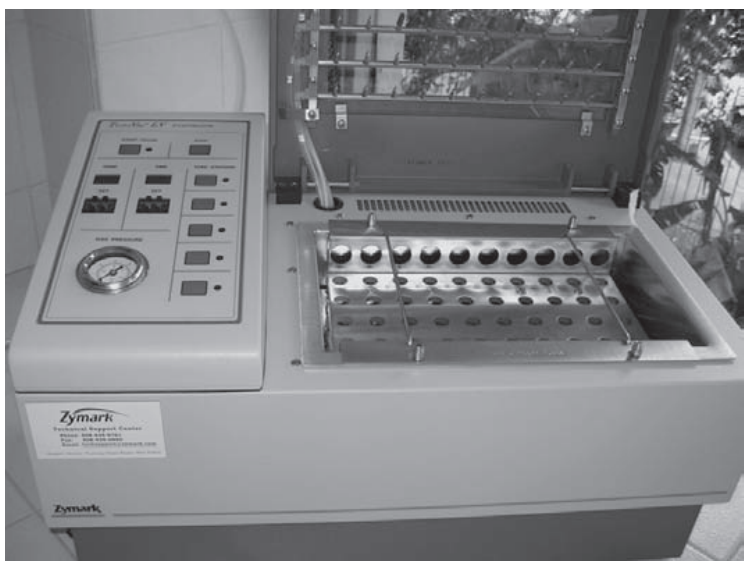

(c)

Figura 5 En muchos extractos obtenidos, los analitos se encuentran en concentraciones por debajo de los niveles mínimos de detección o cuantificación de las técnicas cromatográficas GC ○ GC-MS empleadas y requieren, previo a su análisis, la concentración. a) Dispositivo sencillo, "manual" para la concentración de la muestra con flujo de gas inerte (por lo común, nitrógeno). b) Concentrador tipo Kuderna-Danish, donde generalmente se concentran extractos obtenidos por las técnicas de extracción líquido-líquido (LLE) en continuo o Soxhlet. c) Evaporador automático provisto de controles de flujos de gases, temperatura y tiempo del proceso de evaporación-concentración.

de automatizar el proceso e identificar químicamente la estructura de los analitos aislados y cuantificarlos reproduciblemente.

Previo a su análisis, es importante conservar correctamente tanto a la muestra, como a los extractos obtenidos. Según el tipo y el origen de 
la muestra (agua, suelo, alimento, tejido vegetal o animal, fluido fisiológico, etc.), existen diferentes protocolos y normas (acorde con las agencias reguladoras, EPA, ASTM, AOAC, etc.) para su conservación adecuada, el tipo de recipiente a usar, la temperatura, humedad y luz o la necesidad de la adición de algunas sustancias (conservantes, antioxidantes) o realización de procedimientos adicionales (agregación de sales o solventes, cambio de $\mathrm{pH}$, temperaturas muy bajas, etc.), que impiden el deterioro de la muestra, e.g., su oxidación, hidrólisis o la formación de artefactos.

La muestra, incorrectamente guardada, puede experimentar cambios en su composición y producir pérdidas de algunos analitos de interés. Esto puede acontecer a razón de algunos cambios físicos (evaporación, precipitación, difusión, percolación, adsorción, etc.) y químicos (oxidación, reducción, hidrólisis, degradación microbiana, fotólisis, etc.) y conducir a los falsos negativos (por ejemplo, la conversión de fenoles, inicialmente presentes en la muestra, a ácidos) o a los falsos positivos (formación de artefactos, e.g., "aparición" de algunas sustancias controladas, que no estaban presentes inicialmente en la muestra y se formaron durante su almacenamiento, por ejemplo, hidrocarburos aromáticos $\rightarrow$ fenoles). El material del empaque, los recipientes y las tapas pueden retener algunos analitos (por ejemplo, adsorción de hidrocarburos poliaromáticos en la superficie de vidrio) o, al contrario, "aportar" al extracto sustancias (aditivos, colorantes, ftalatos, etc.), que no estaban presentes inicialmente en la muestra original.

Un ejemplo curioso aparece registrado en la Figura 6. Hace unos años, en nuestro laboratorio,

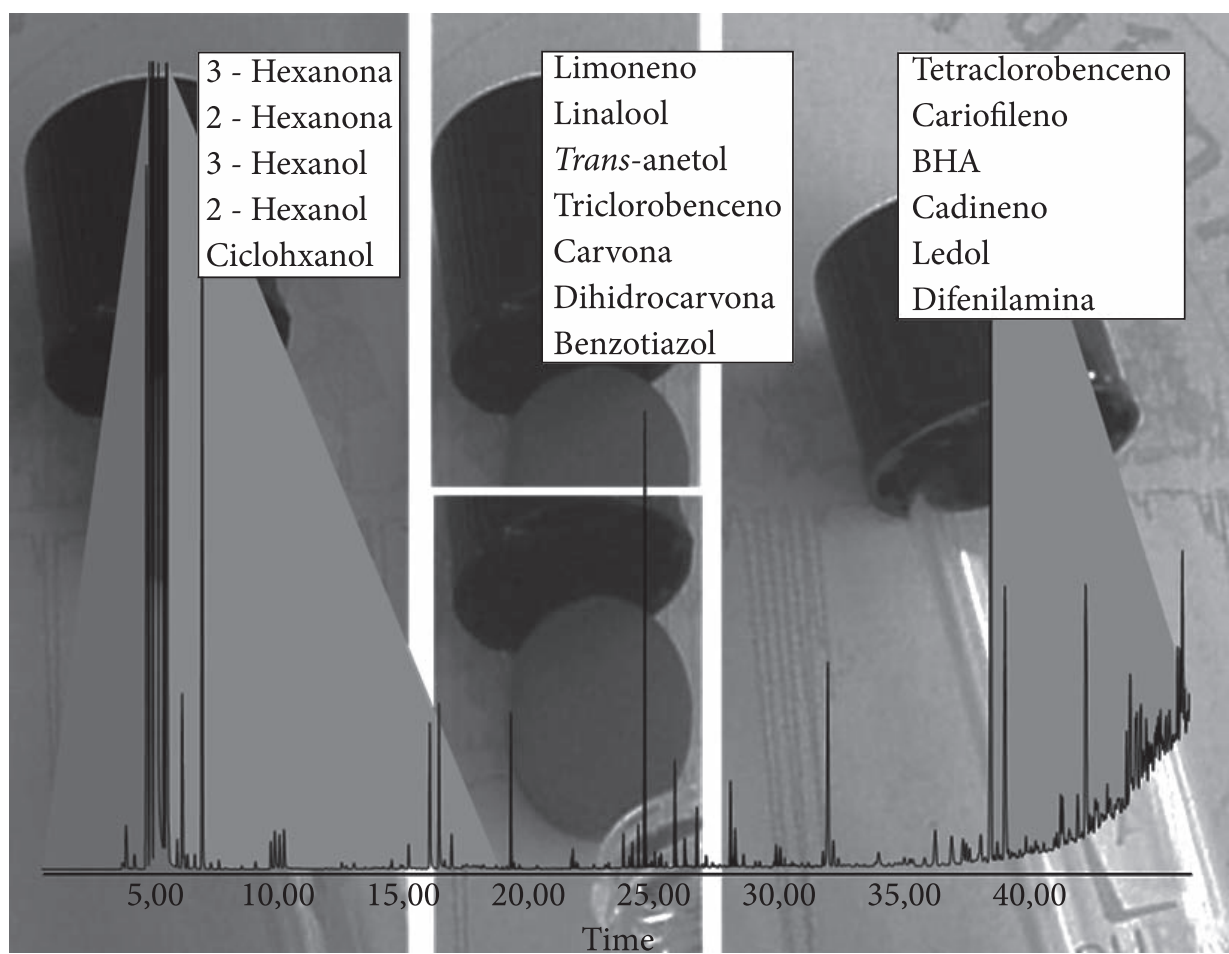

Figura 6 Cromatograma del extracto de las tapas de tubos de ensayo, obtenido por extracción con hexano caliente y su posterior concentración con flujo de $\mathrm{N}_{2}$. Se observan compuestos traza presentes en el disolvente hexano "concentrado" y los compuestos que estuvieron en contacto con las tapas durante la manipulación o almacenamiento de muestras. Las tapas pueden revelar la "historia" química del laboratorio y de los analitos que en él se manejaban. GC-MS (El, 70 eV). Columna DB-5 (60 m). Inyección (1 $\mu \mathrm{L})$ en el modo split (1:30). 
donde se llevaban a cabo varios trabajos de grado, se usaban y se re-usaban tubos de ensayo con sus respectivas tapas. Los sellos de caucho de estas tapas se sometieron a la extracción con hexano caliente y el extracto obtenido, que se concentró casi a sequedad con un flujo de nitrógeno seco, se inyectó al equipo GC-MS. En el cromatograma resultante es posible leer la "historia" del laboratorio, a través de las sustancias impregnadas en la tapas plásticas y extraídas de ellas. Las hexanonas, hexanoles y ciclohexanol fueron impurezas del hexano y "aparecieron" cuanto este solvente se concentró varias veces (se hizo blanco del disolvente concentrado); los terpenoides (linalool, cariofileno, ledol, otros) provenían de varios trabajos realizados en el área de fitoquímica, los clorobencenos provenían de los estudios de aceites dieléctricos (PCBs) y el benzotiazol y la difenilamina fueron, respectivamente, el agente derivatizante y el patrón interno, usados en los estudios de antioxidantes y para la cuantificación de fármacos. Para los servicios analíticos es menester, para evitar la contaminación cruzada y los efectos de carry-over, usar el material (e.g., tapas) desechable. Esto, por supuesto, eleva bastante el costo de los análisis.

Igualmente, es muy importante tener precaución cuando se guardan los extractos -ya obtenidos-, en frascos (viales), para su posterior análisis por GC o GC-MS. El almacenamiento prolongado del extracto en disolvente, e.g., diclorometano, puede conducir a que a éste pasen los compuestos, productos de la degradación del septum de la tapa, y en el cromatograma del extracto se observan entonces los picos intrusos, cuya presencia puede no permitir la cuantificación correcta de analitos o generará la superposición de las señales. En la Figura 7, se observa otro ejemplo, en el cual se comparan las fracciones volátiles de una bebida tipo Cola, obtenidas por dos técnicas de extracción, una solventless,

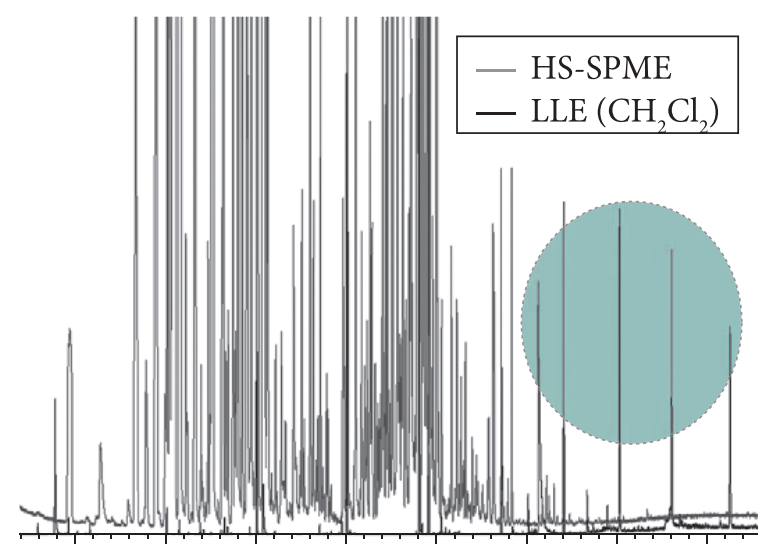

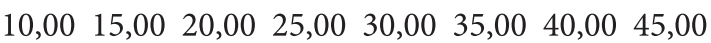

Figura 7 Cromatogramas de: 1) la fracción volátil obtenida por HS-SPME (fibra PDMS/DVB, $65 \mu \mathrm{m}$ ) de la bebida tipo Cola (línea verde) y 2) el extracto de la misma bebida obtenido por el método LLE por lotes, con diclorometano (línea negra). En el extracto LLE aparecen (resaltadas con un círculo) sustancias "intrusas" (productos de degradación de la silicona del septum de la tapa, co-extraídos por el $\mathrm{CH}_{2} \mathrm{Cl}_{2}$ durante el almacenamiento de la muestra). Se observa, que la extracción de los analitos volátiles de la bebida por LLE fue bastante deficiente, en comparación con el método HS-SPME. GC-MS (El, 70 eV). Columna DB-5 (60 m).

micro-extracción en fase sólida en modo headspace, HS-SPME, y la otra, extracción líquidolíquido, LLE, por lotes, usando diclorometano. La última, además de resultar menos sensible y contaminante, presentó problemas de co-extracción de productos de degradación de un septum de silicona de la tapa del frasco donde se almacenó el extracto, antes de su inyección al GC-MS.

Uno de los procedimientos fundamentales en la cadena analítica y de preparación de la muestra es el análisis de blancos. Éstos se dividen en blancos positivos (cuando se utiliza la muestra "simulada" enriquecida con el analito de interés, añadido a la matriz en cantidad conocida), y blancos negativos [una muestra (matriz) desprovista de analito-target]. Los blancos positivos demuestran que el método de extracción funciona y es reproducible y cuantitativo. Los blancos negativos son obligatorios para demos- 
trar que el procedimiento empleado es limpio, libre de la posible contaminación cruzada proveniente de material de vidrio, manos, tapas, mangueras, jeringas, disolventes, viales, septa u otras partes del instrumento analítico (GC, GC-MS), e.g., inyector, línea de transferencia, cámara de ionización, columna, etc., que están involucrados en el análisis y están en contacto con la muestra. Para demostrar que el equipo GC-MS no posee efectos de memoria (carry-over), entre los extractos -que se analizan-, se intercalan los disolventes puros, cada 3-5 muestras o más frecuentemente, si el análisis es de trazas y cuando es particularmente crítico el resultado obtenido (análisis ambiental, forense, etc.).

El principal propósito de la preparación de la muestra es el aislamiento con alta eficiencia (recuperación) de los analitos de interés. Pero, ¿cómo se sabe que el proceso de extracción es eficiente, cuantitativo y reproducible? Para ello, se utilizan diferentes estrategias, pero, muy a menudo, se emplea un testigo, una sustancia surrogate, es decir, un compuesto sucedáneo con características físico-químicas similares a las de los componentes-target a extraer. El surrogate debe ser una sustancia pura (material de referencia certificado), estructuralmente similar al compuesto o compuestos a aislar y cuantificar, no debe estar presente en la muestra, ni reaccionar con ninguno de sus componentes. Es una sustancia similar a un patrón interno, pero se agrega a la muestra antes de someterla a la extracción. Desde el punto de vista químico y analítico, los mejores surrogates son análogos deuterados de los analitos-target, aunque no siempre es posible conseguirlos. La ventaja de un análisis por GC-MS de un extracto enriquecido con la sustancia surrogate (análogo deuterado), es que el surrogate y el (los) analito(s)-target pueden ser analizados y cuantificados simultáneamente y sin la necesidad de que sus picos cromatográficos se separen en la columna (de hecho, co-eluyen). Por ejemplo, para cuantificar naftaleno (P.M. 128) en una muestra, se puede usar como surrogate su deuteroanálogo (P.M. 136). El análisis cuantitativo del naftaleno se basa en las mediciones de las corrientes iónicas parciales de iones moleculares de naftaleno $\left(\mathrm{M}^{+}, m / z 128\right)$ y de su análogo deuterado $\left(\mathrm{M}_{\mathrm{d} 10}{ }^{+}, m / z 136\right)$. El cálculo del porcentaje de recuperación con base en las cantidades del surrogate añadido $\left(\mathrm{C}_{\text {teor }}\right)$ y extraído $\left(\mathrm{C}_{\exp }\right)$, se hace como se ilustra en la Figura 8. Sin embargo, es importante tener en cuenta que la incorporación de la sustancia surrogate en la muestra real no siempre refleja la misma manera en la cual los analitos-target fueron incorporados en la matriz; por ello, los porcentajes de recuperación de las sustancia(s)-surrogate pueden ser más altos que los de los analitos-target, sobre todo, cuando la matriz es heterogénea y muy compleja (suelo, lodo, tejido vegetal, roca, etc.).

La cuantificación de analitos-target se basa en la curva de calibración, que puede ser obtenida tanto por el método de estandarización

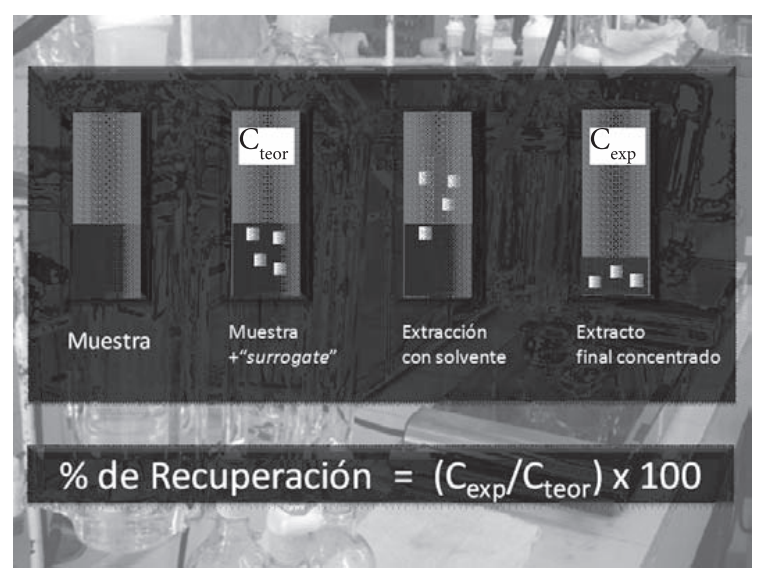

Figura 8 Proceso esquemático de la adición de una sustancia-surrogate a la muestra (matriz), que luego se somete al proceso de extracción. La cantidad de surrogate extraída, con respecto a la añadida, permite calcular el porcentaje de recuperación de analito(s)target, estructuralmente similar(es) a la molécula del surrogate. 
externa, como interna, usando un compuesto estándar (Istd, Internal Standard), que se agrega al extracto final concentrado, para cuantificar tanto a los analitos-target, como a la sustanciasurrogate recuperada, para hallar el porcentaje de recuperación del surrogate y, por consiguiente, de los analitos.

Entre los principales problemas comunes, que acompañan a diferentes métodos de preparación de la muestra, figuran el tiempo muy prolongado que requiere esta etapa (60-85\% del tiempo total), su elevado costo (materiales, disolventes, patrones, personal, etc.), la eficiencia de recuperación de analitos de la matriz, a menudo, muy pobre y una baja reproducibilidad, así como la probabilidad de contaminación cruzada y errores en la interpretación de resultados. Las fuentes más frecuentes de la contaminación cruzada son los reactivos, agentes derivatizantes, disolventes, material de vidrio, tapas, mangueras, pipetas, manos (sic!), atmósfera del laboratorio, entre otras. El respectivo blanco del procedimiento permite garantizar que el proceso de preparación de la muestra está libre de la contaminación cruzada. Para ello, se lleva a cabo la preparación de la muestra, i.e., extracción, a partir de la matriz simulada sin analito(s)-target. Por ejemplo, se realiza el análisis de disolvente concentrado, que permite vislumbrar tanto sus impurezas, como los posibles artefactos provenientes del material de vidrio, pipetas, columnas de limpieza, etc.; para las técnicas de extracción en fase vapor (headspace) es importante obtener el blanco de la atmósfera del laboratorio, así como realizar una adquisición de datos en el equipo GC-MS, pero sin inyectar ninguna muestra, para descartar los efectos carry-over del aparato.

Se puede concluir, resumiendo, que entre los puntos clave de la preparación de la muestra figuran la eficiencia de extracción, la calibración con patrones para la cuantificación correcta de analitos, los análisis de blancos de procedimiento (tanto positivos, como negativos), el uso del material certificado de referencia, el análisis de duplicados, la verificación sistemática de la curva de calibración, la debida calificación del operador y técnico del laboratorio, la bitácora y el registro minucioso de todo el acontecimiento analítico, entre otros aspectos.

\section{Métodos de extracción más comunes en la preparación de la muestra para el análisis por GC-MS}

En los últimos años el desarrollo de diferentes técnicas de extracción y de preparación de la muestra ha experimentado un incremento excepcional, gracias a su importancia para el análisis instrumental. En la Figura 9 se puede observar este crecimiento, sobre todo, en la última década. Las revistas donde más se publican los resultados de investigación que involucran distintas técnicas de extracción son, en su orden decreciente, las siguientes: Journal of Chromatography A, Analytica Chimica Acta, Analytical Chemistry, Journal of Agricultural and Food Chemistry, entre otras. Es interesante anotar, que las áreas donde más se desarrollan y aplican las diferentes técnicas extractivas son química, bioquímica, análisis biológico, agrícola y ambiental, entre otras (Véase Figura 10).

La escogencia correcta de la técnica de extracción depende de muchos factores. Entre éstos, figuran: 1) la naturaleza de la matriz (origen, estado de agregación, homogeneidad, estabilidad y representatividad) y del analito a aislar (su volatilidad, polaridad, reactividad y termoestabilidad, en qué concentración se encuentra y cómo está distribuido en la matriz); 2) el propósito mismo del análisis, i.e., cualitativo o cuantitativo; 3) la necesidad de la confirmación de la estructura química del analito (identificación 


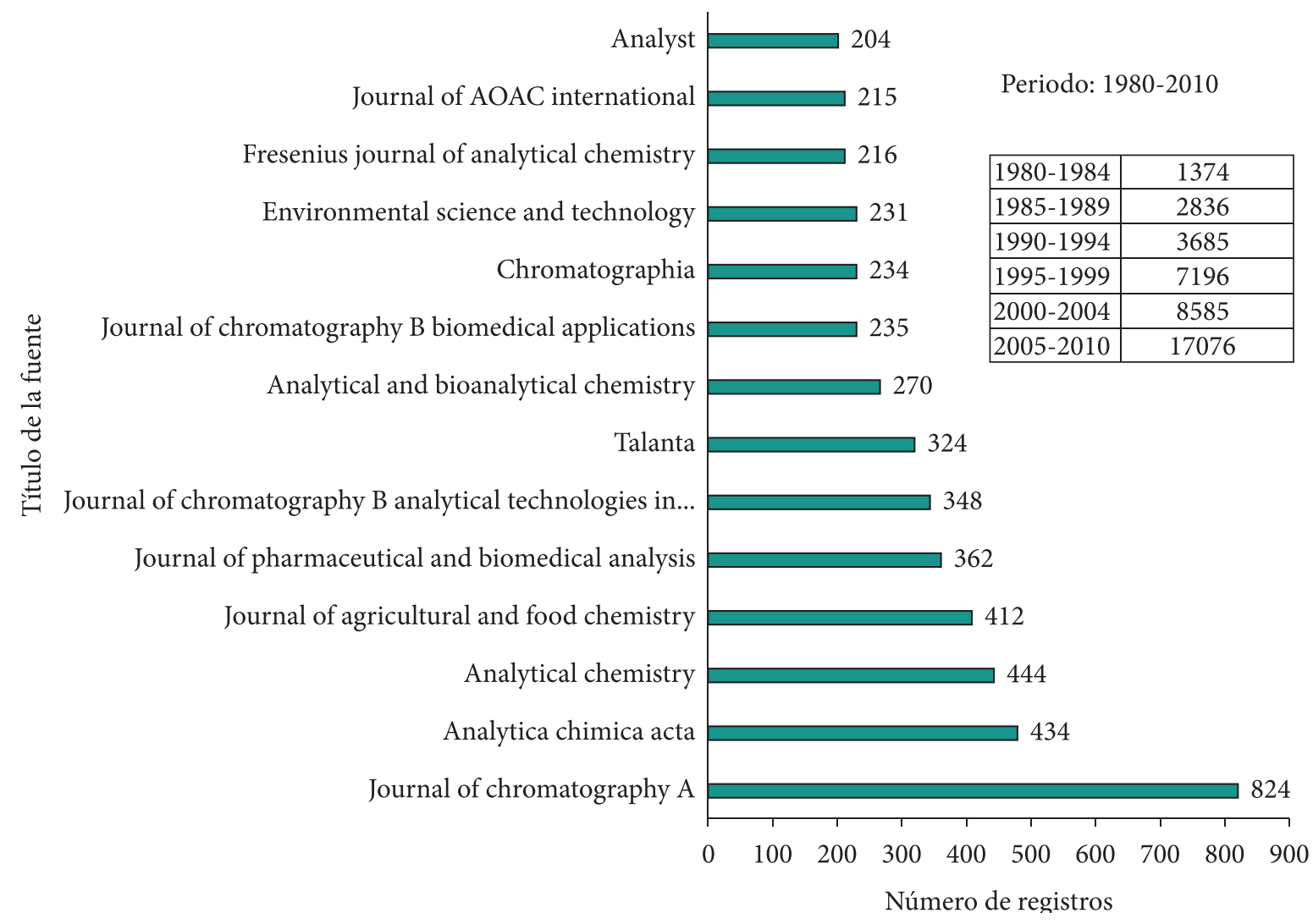

Figura 9 Desarrollo cronológico de publicaciones científicas sobre los métodos de extracción para análisis instrumental. Las principales revistas donde se publica sobre este tópico. Base de datos: Scopus (Elsevier, 2011 ). Período de observación: 1980-2010. Palabras-clave: "Extraction techniques" OR "Analytical method". Fecha de consulta: 19 de enero de 2011.

por técnicas espectroscópicas); 4) la premura del análisis, es decir, qué tan urgente es el resultado (por ejemplo, para determinar el tipo de veneno o sustancia tóxica en el jugo gástrico, para aplicarle rápido un antídoto correcto a un paciente intoxicado); 5) las implicaciones legales que pueden representar los resultados de análisis obtenidos (por ejemplo, en campos forense, ambiental, control de doping) y, por último, 6) es importante saber si el método de extracción es conocido, regulado o debe ser establecido, optimizado y validado. Para los análisis de extractos por GC-MS, lo más importante es tener el extracto "limpio" (sin componentes no compatibles con esta técnica) y suficientemente concentrado, para que los analitos se encuentren en él en la concentración conmensurable con los niveles mínimos de detección/cuantificación del sistema GC-MS respectivo.

Los métodos de extracción de los analitostarget pueden dividirse grosso modo en tres grupos grandes, a saber: I. Métodos Destilativos. II. Métodos Extractivos. III. Métodos de Headspace. Algunas técnicas sui generis pueden combinar dos métodos de diferentes grupos, por ejemplo, destilación-extracción simultánea con solvente ${ }^{50}$ (SDE, Simultaneous Distillation-Extraction, por sus siglas en inglés). Las técnicas de destilación están basadas en las diferencias (deben ser grandes) de las propiedades físico-químicas de los analitos a aislar y de la matriz; concretamente, sus temperaturas de ebullición (volatilidades o 


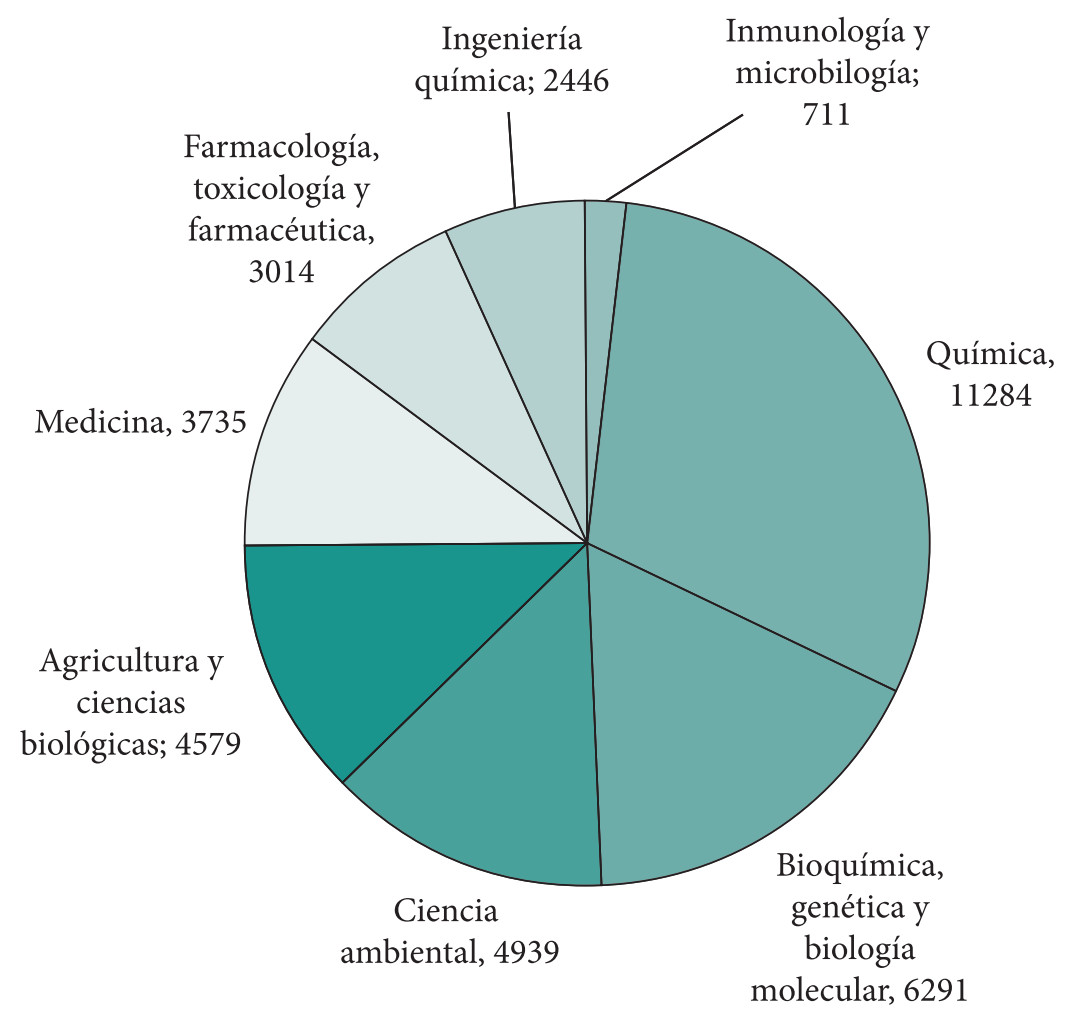

Figura 10 Principales áreas de aplicación de diferentes técnicas de extracción empleadas previamente al análisis instrumental. Base de datos: Scopus (Elsevier, 2011). Período de observación: 1980-2010. Palabrasclave: "Extraction techniques" OR "Analytical method". Fecha de consulta: 19 de enero de 2011.

presiones de vapor). La fracción de interés podría ser tanto la que se destila (volátil), como el remanente (fondo de destilación). Para el análisis por GC o GC-MS se utiliza comúnmente la fracción volátil. Las destilaciones pueden llevarse a cabo de diferentes maneras: 1) Arrastre con vapor (SD, Steam Distilllation, pos sus siglas en inglés); 2) Hidrodestilación (HD, Hydrodistillation, por sus siglas en inglés) o 3) Destilación con aguavapor. Véase la Figura 11 para diferenciar los tres métodos que se utilizan fundamentalmente para la destilación del material vegetal y obtención de aceites esenciales o, en algunas aplicaciones, para el aislamiento de fracciones volátiles de productos finales (jabones, champús, algunas bebidas, cremas dentífricas, etc.). Los extractos o fracciones volátiles destilados por $\mathrm{SD}, \mathrm{HD}$ o SDE presentan mezclas perfectas para su análisis por GC-MS. Sólo pueden requerir un secado, e.g., con sulfato de sodio anhidro, previo a la inyección del extracto al cromatógrafo. Algunos procesos de destilación suceden a presión reducida (vacío), en columnas especiales (destilación molecular); muchos se realizan a escala industrial; pero, en general, no son aptos para estudiar y aislar compuestos a nivel de trazas.

Con miras a aumentar la eficiencia de extracción y reducir sustancialmente el tiempo de destilación, en los últimos 25 años se emplea activamente la radiación de microondas como fuente de calentamiento ${ }^{51-53}$, tanto del agua (hidrodestilación asistida por microondas, MWHD, Microwave-Assisted Hydrodistillation, por sus siglas en inglés), como de disolvente o de la muestra, según las constantes dieléctricas, $\varepsilon$, del uno o del otro (para que la sustancia absorba la radiación de microondas, debe poseer $\varepsilon$ alta, 

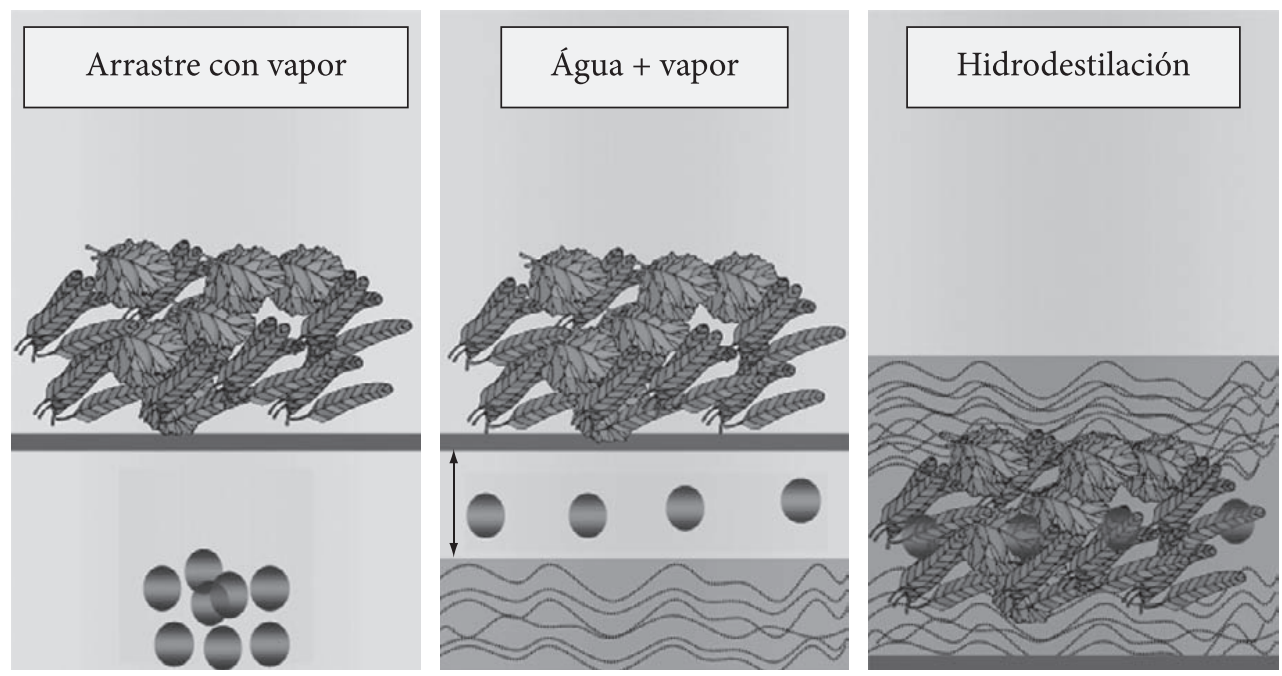

Figura 11 Obtención de aceites esenciales por arrastre con vapor (fuente externa de vapor, e.g., caldera); destilación con agua-vapor (vapor húmedo obtenido por calentamiento del agua hasta su vigorosa ebullición) e hidrodestilación (calentamiento del agua con el material vegetal sumergido, generalmente muy delicado, por ejemplo, flores).

i.e., momento dipolar), formando un grupo de métodos de extracción asistida por radiación de microondas (MAE, Microwave-Assisted Extraction, por sus siglas en inglés). El método MAE se utiliza más que todo para la extracción de muestras sólidas (material vegetal, suelo, tejidos, etc.) con agua o con un disolvente orgánico $^{53,54}$.

La segunda familia de técnicas, comprende diversos métodos extractivos, en los cuales el aislamiento de analitos está basado en diferencias de sus solubilidades en disolvente(s) o de su capacidad de adsorción o absorción sobre un sorbente (carbón activado, gel de sílice, alúmina, tamiz molecular, etc.) o en un polímero poroso (PDMS, Tenax, Porapak, Chromosorb, resinas sintéticas, etc.). Entre los métodos extractivos "clásicos" figuran, la extracción líquido-líquido, LLE (Liquid -Liquid Extraction, por sus siglas en inglés), en modo continuo o por lotes (batch). La selectividad de extracción se logra con una escogencia correcta del disolvente (polaridad, temperatura de ebullición, constante dieléctrica, capacidad de formar puentes de hidrógeno, dis- ponibilidad, accesibilidad, costo, etc.); a menudo, el extracto obtenido debe someterse a los procesos de limpieza y concentración. A pesar de una serie de problemas que acarea la técnica de LLE, entre otros, la formación de emulsiones, alto costo, tiempos prolongados de extracción, dificultad de automatización, disposición de disolventes tóxicos, posibilidad de contaminación cruzada, etc., en la última década, se observa un número creciente de trabajos publicados que involucran el uso de LLE (Figura 12).

Como una alternativa a la LLE, desde hace ca. 40 años, se ha implementada la extracción en fase sólida, SPE (Solid-Phase Extraction, por sus siglas en inglés) ${ }^{55,56}$, que permite reducir sustancialmente el uso de disolventes y combinar en un solo paso la extracción, limpieza y concentración de analitos-target; además; el método incluye la posibilidad de la automatización, implementada en varios equipos comerciales con conexión en línea al cromatógrafo. Los datos estadísticos muestran el crecimiento de la aplicación de este método de preparación de la muestra en diferentes áreas de investigación (Figura 12). 

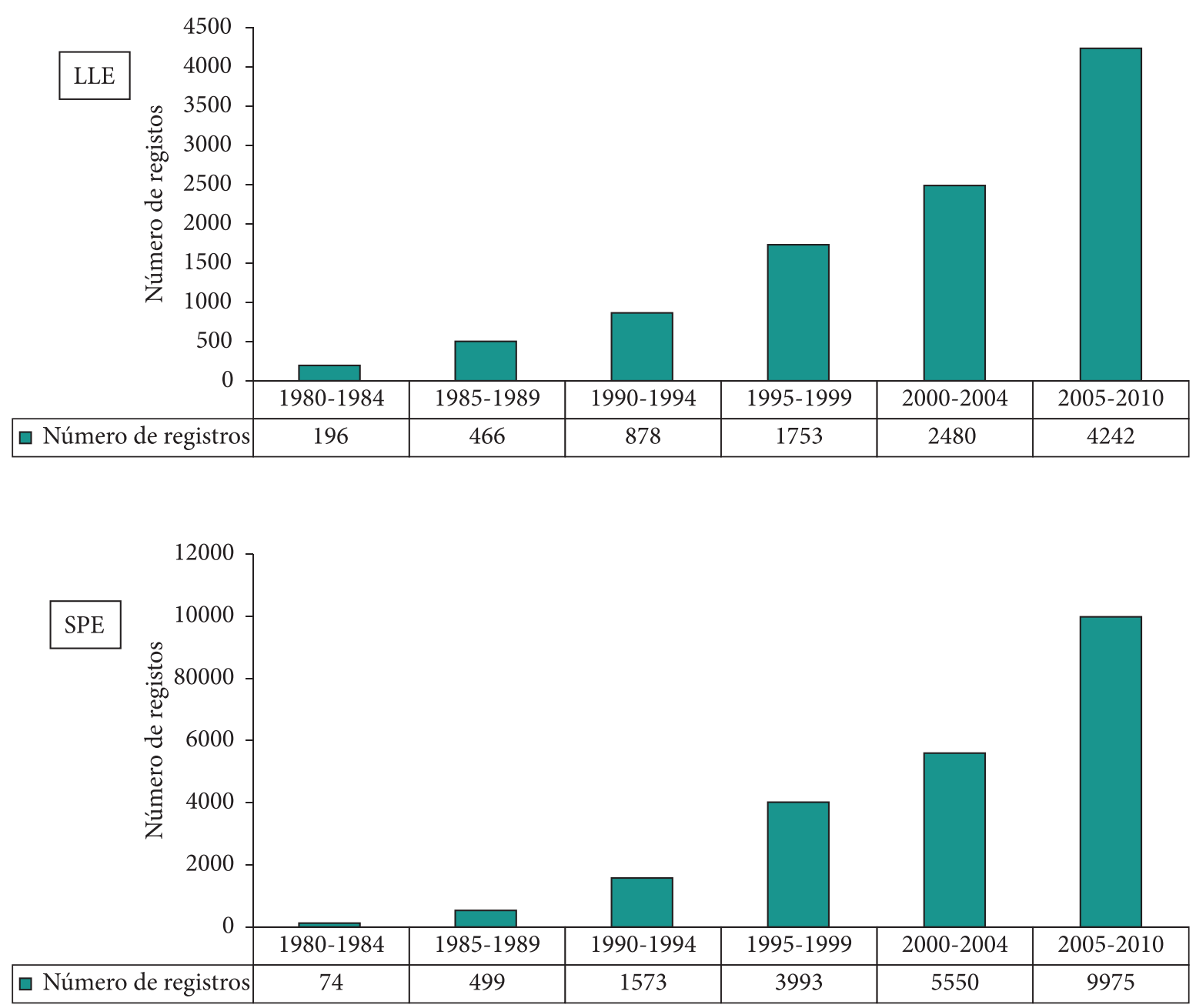

Figura 12 Crecimiento en función del tiempo de publicaciones científicas que involucran la utilización de técnicas extractivas de preparación de la muestra, i.e., extracción líquido-líquido (LLE) y en fase sólida (SPE). Base de datos: Scopus (Elsevier, 2011 ). Período de observación: 1980-2010. Palabras-clave: "Liquid-Liquid Extraction" y "Solid-Phase Extraction", respectivamente. Fecha de consulta: 19 de enero de 2011.

La extracción con fluido supercrítico, SFE (Supercritical Fluid Extraction, por sus siglas en inglés), a pesar del costo alto en comparación con otras técnicas, en los últimos diez años ha experimentado un intenso crecimiento (Figura 13). SFE se ha usado en las áreas de productos naturales, ambiental, alimentos, forense y en muchas aplicaciones industriales. La selectividad del método SFE se alcanza variando los parámetros operacionales, fundamentalmente, de la densidad de fluido supercrítico (presión y temperatura), así como usando modificadores ${ }^{57-60}$. Para el análisis por GC-MS, a menudo, se requiere la limpieza adicional del extracto SFE, para eliminar grasas o pigmentos, ceras u otros compuestos de alto peso molecular, según la naturaleza de la matriz que se usó.

Entre los métodos extractivos más antiguos (centenario, sic!), pero tal vez, menos selectivos y más "sucios" -en cuanto al extracto que se obtiene-, figura la Extracción Soxhlet, que se usa en los análisis de suelos, polímeros, productos naturales, etc., en áreas de bioquímica, ciencias agrícolas, biológicas, ambientales, etc. (Figura 14), pero no presenta el crecimiento tan 


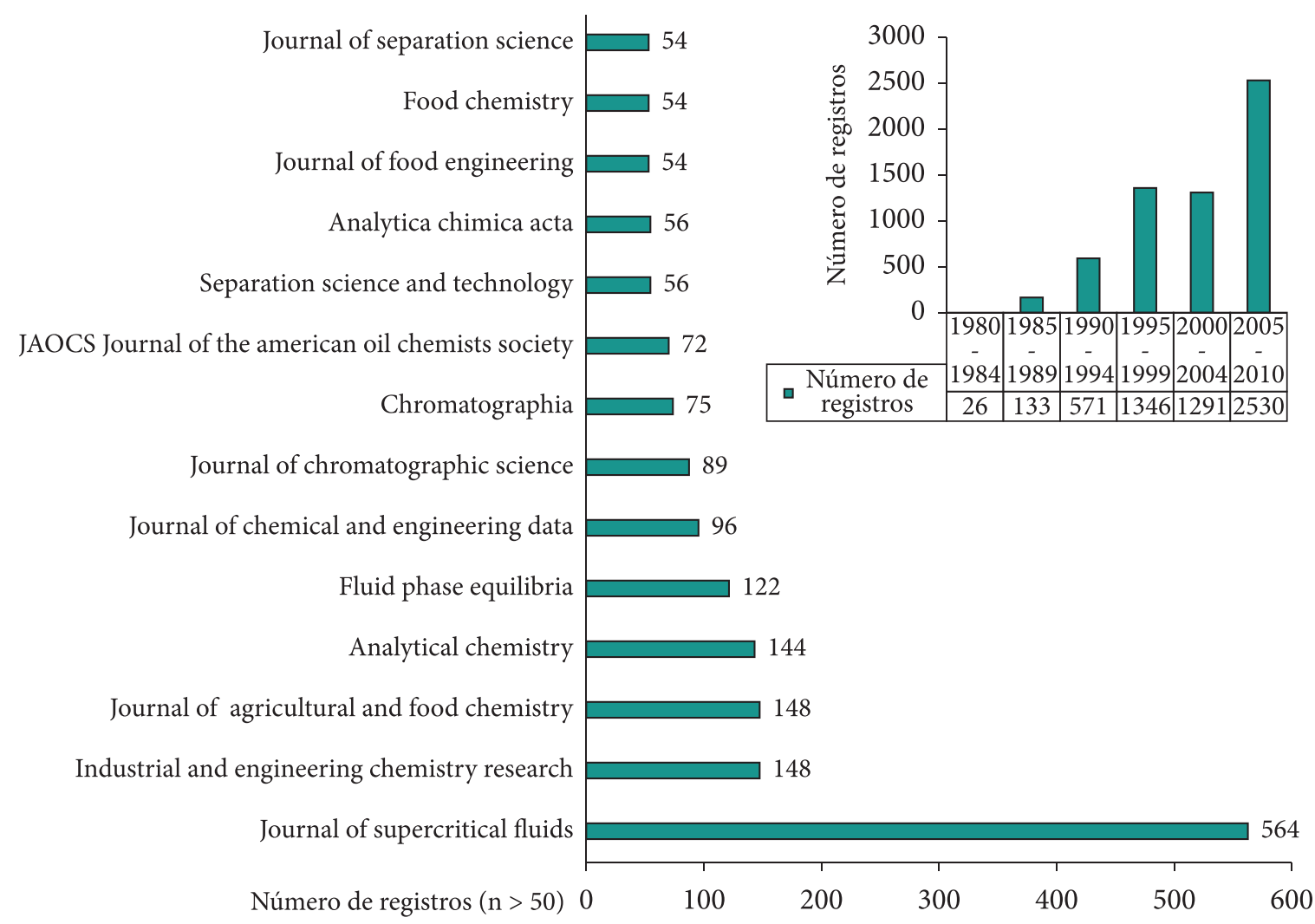

Figura 13 Desarrollo cronológico de publicaciones científicas sobre el método de extracción con fluido supercrítico, SFE. Las principales revistas donde se publica sobre este método. Base de datos: Scopus (Elsevier, $2011)$. Período de observación: 1980-2010. Palabras-clave: "Supercritical Fluid Extraction". Fecha de consulta: 19 de enero de 2011.

rápido que ostentan otros métodos, e.g., MAE, SFE, SPME, entre otros, y que se reemplaza frecuentemente, hoy en día, por el método más eficiente, de la Extracción con Solvente Acelerada, ASE (Accelerated-Solvent Extraction, por sus siglas en inglés) $)^{61,62}$, donde la mayor selectividad, rapidez y la considerable reducción del tiempo y de la cantidad de disolvente se logran en un solo paso de extracción, concentración y limpieza, en modo automático y con posibilidad de acoplamiento en línea con GC-MS.

Si se habla de una verdadera "revolución verde" en las técnicas de preparación de la muestra, esta será la Microextracción en Fase Sólida, SPME, que en los últimos ca. 20 años, logró tener un crecimiento sin parangón (Figura 15), gobernar las áreas de análisis de volátiles de origen bio- lógico, estudios ambientales, forenses (acelerantes de incendios, residuos de explosivos, análisis de drogas en fluidos y tejidos), alimentos, monitoreo de diferentes procesos in vitro e in vivo (análisis de aliento, procesos de fermentación, cambios microbiológicos, farmacocinética, etc. ${ }^{63-66}$. La SPME combina la extracción y concentración de analitos-target $\mathrm{y}$ puede usarse en tres modos: 1) en headspace, 2) por sumergimiento directo de la fibra en la muestra y 3) usando una membrana protectora. Es una técnica amena al análisis de compuestos por GC-MS, más aún que en el mercado existen los dispositivos que permiten realizar este proceso de modo automático y acoplado en línea con el cromatógrafo (Figura 16). La selectividad del método SPME se logra: 1) variando las condiciones operacionales (tem- 


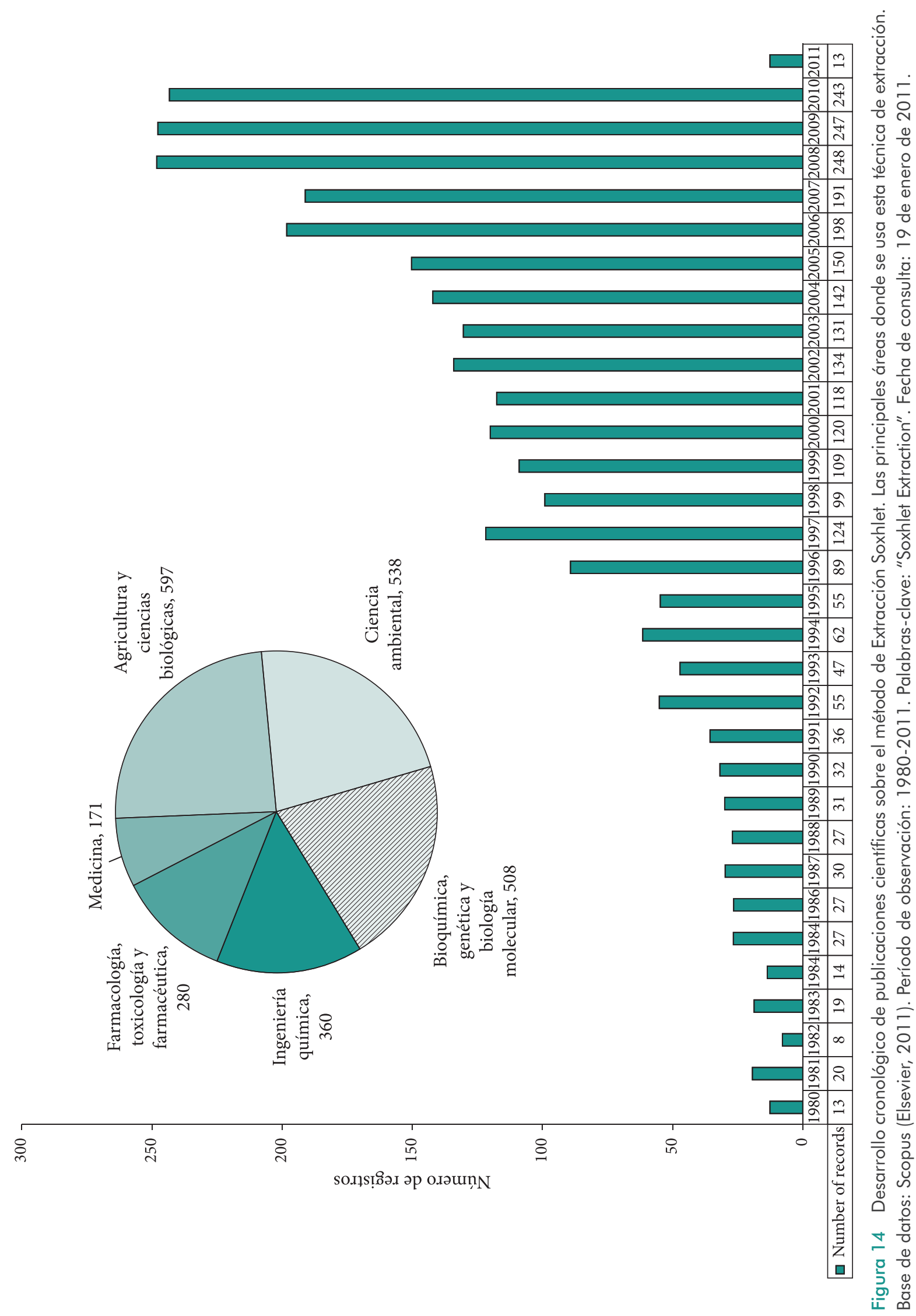




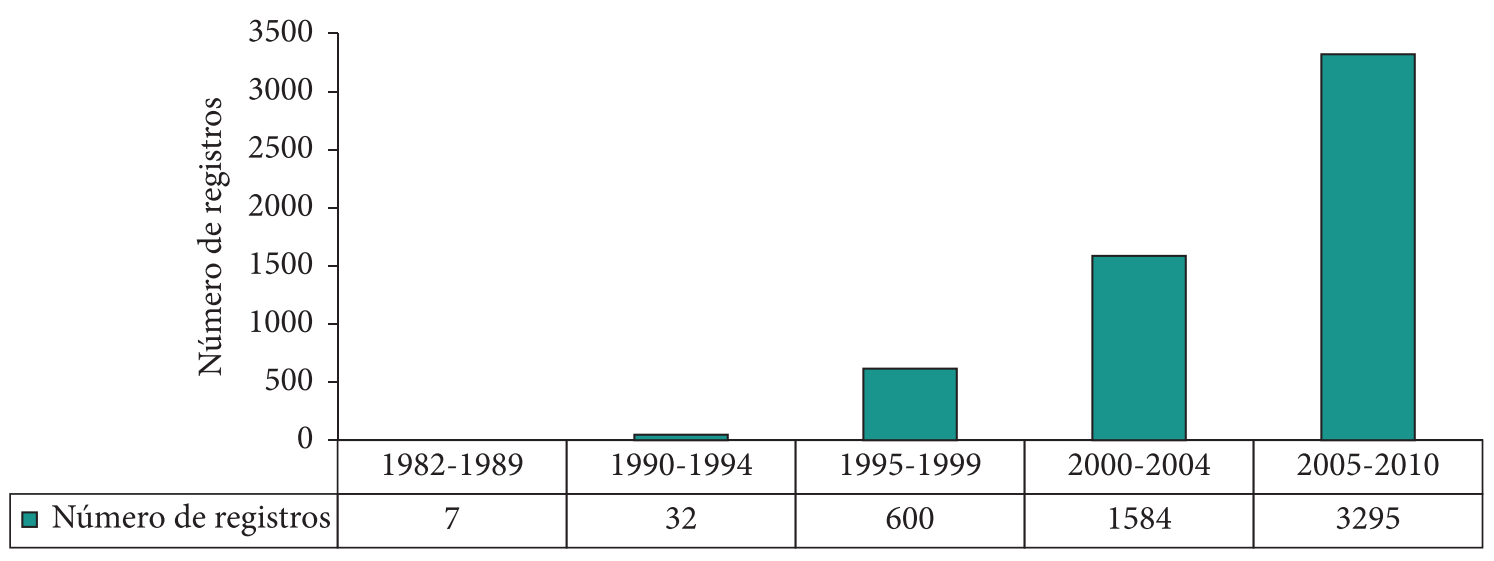

(a)

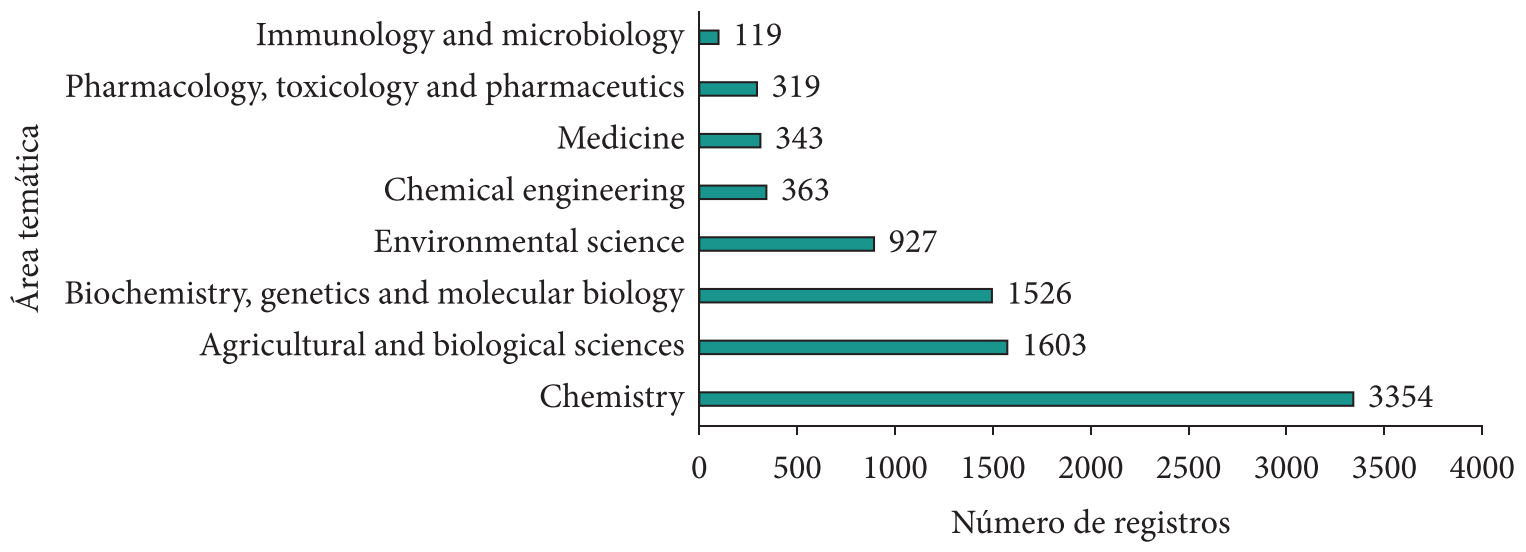

(b)

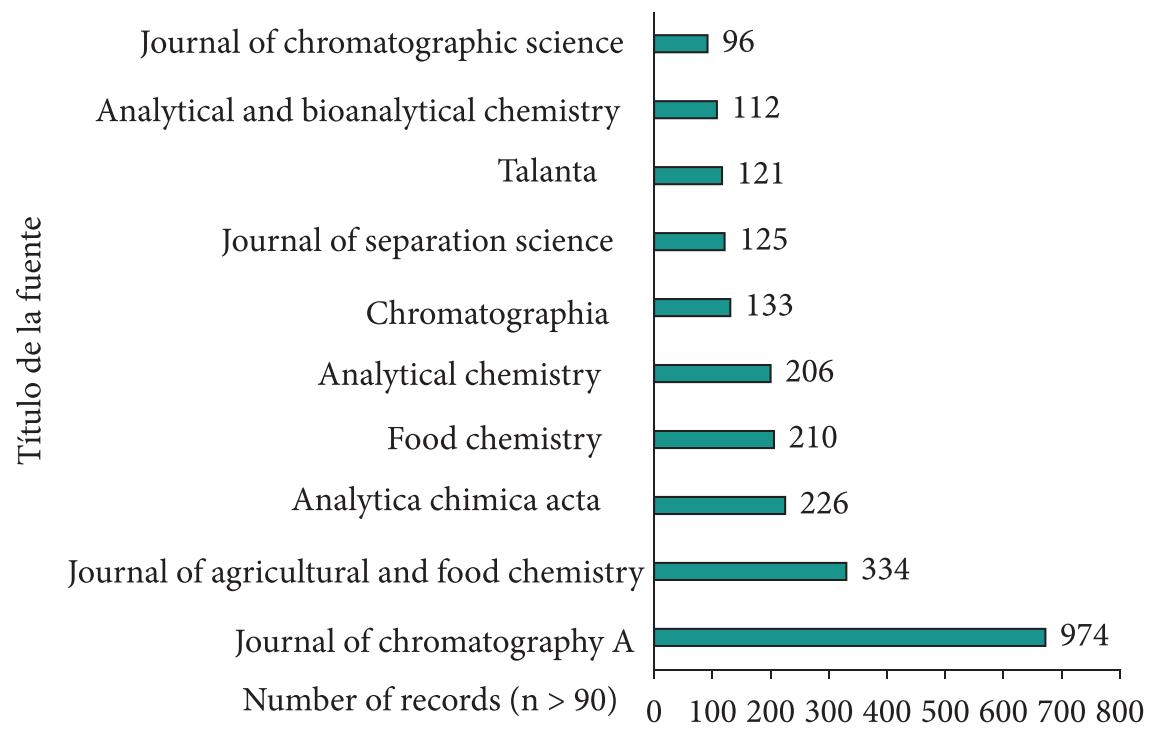

(c)

Figura 15 a) Desarrollo cronológico de publicaciones científicas que incluyen el método de extracción SPME. b) Las principales áreas de investigación donde se usa SPME. c) Revistas principales donde más se han publicado trabajos que emplean SPME. Base de datos: Scopus (Elsevier, 2011). Período de observación: 1980-2010. Palabras-clave: "SPME". Fecha de consulta: 19 de enero de 2011. 


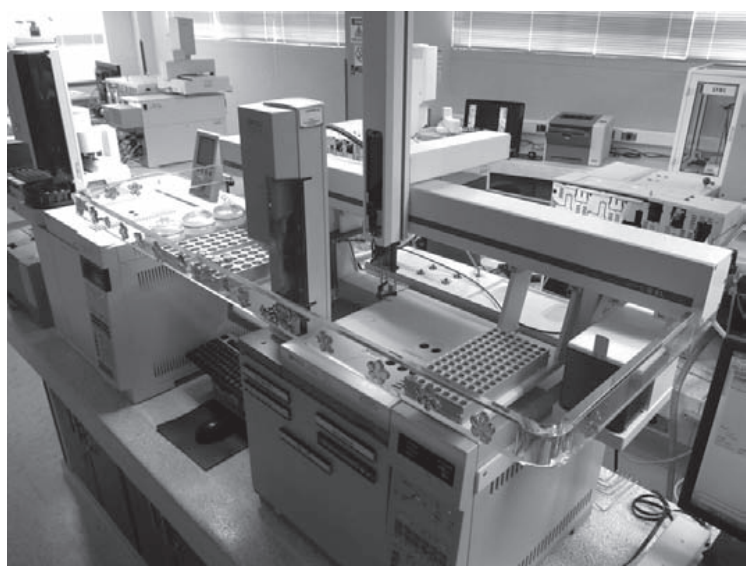

Figura 16 Cromatógrafo de gases acoplado a un sistema de preparación de muestras con SPME automatizado.

peratura, salting-out, modo de exposición de la fibra, agitación y el tipo de recubrimiento de la fibra, entre otros; 2) usando sistemas de detección cromatográfica selectivos (ECD, FPD, NPD, MS-SIM, etc.) y 3) aprovechando la derivación de los analitos-target en matriz o directamente sobre la fibra ${ }^{67,68}$. Una de la aplicaciones más interesantes de SPME es la extracción de analitos-target acompañada de su derivación 1) en la matriz; 2) en la fibra saturada con un agente derivatizante ${ }^{67}$ (Figura 17) o 3) directamente en el puerto de inyección del cromatógrafo.

Los métodos de muestreo de espacio de cabeza (Headspace) en sus versiones "clásicas" se dividen en: (1) Headspace estático (S-HS) y (2) Headspace dinámico. Las fracciones volátiles recogidas son aptas para el análisis GC-MS, ya que la fase vapor no contendrá los componentes de matriz e interferencias no volátiles, muy polares (iónicas) o de alto peso molecular. El S-HS se aplica a las muestras con analitos-target en concentraciones relativamente altas y que no requieren la pre-concentración. Son análisis por GC-FID de BTEX (benceno, tolueno, etilbenceno y xilenos) en agua, la cuantificación de trihalometanos en agua potable, alcoholemia (alcohol en sangre), compuestos volátiles en pin- turas, polímeros y determinación de disolventes residuales en fármacos, hidrocarburos en suelos, análisis de bebidas y perfumes, entre otras muchas aplicaciones ${ }^{69}$. Cuando la concentración de analitos-target es muy baja en la muestra (ppt, ppb), se requiere su enriquecimiento (pre-concentración) y el muestreo se hace en modo de Headspace dinámico. Básicamente, los analitos se recogen, al purgar la fase vapor con un gas inerte, en un solvente, o se "congelan" (crio-atrapamiento) o, se ad(b)sorben en un sorbente (método de purga y trampa, P\&T), del cual se recuperan por la acción de temperatura (Termodesorción, TD $)^{70}$ o se eluyen con solvente.

El uso de la fase vapor por encima de la muestra (headspace) como medio para realizar los procesos extractivos tiene grandes ventajas desde el punto de vista analítico, porque se eliminan muchas interferencias. En el estudio de productos naturales, el muestreo headspace directo ha permitido el estudio in vivo de la fracción volátil alrededor de plantas completas, $\mathrm{o}$ sus partes ${ }^{71}$. Tradicionalmente, se distinguen los métodos estático y dinámico de muestreo, aunque las técnicas de sorpción de gran capacidad desarrolladas en la última década involucran ambos aspectos $^{72}$. El gran desarrollo de la técnica de SPME ha servido para identificar, en sus múltiples aplicaciones, algunos aspectos que pueden mejorarse. Esto ha generado una colección de nuevos métodos de muestreo a micro-escala, que logran una mayor capacidad de concentración de los analitos de interés y tal como la SPME, son susceptibles de automatización. In varios casos, se logra una capacidad de concentración superior a la de SPME porque se emplea un volumen mayor de fase sorbente (polimérica, normalmente). En la técnica de trampa de adsorción dentro de la jeringa (INCAT, Inside Needle Capillary Adsorption Trap por sus siglas en inglés), una capa de polímero recubre el interior de la aguja 


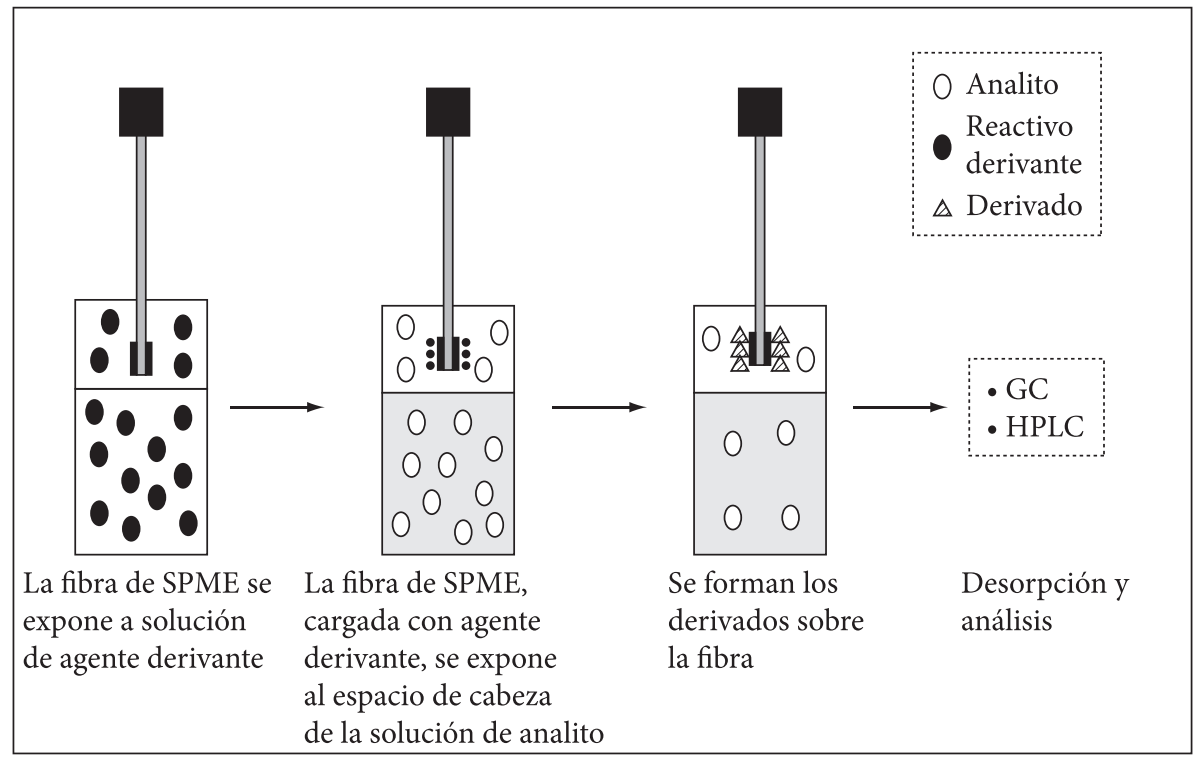

(a)

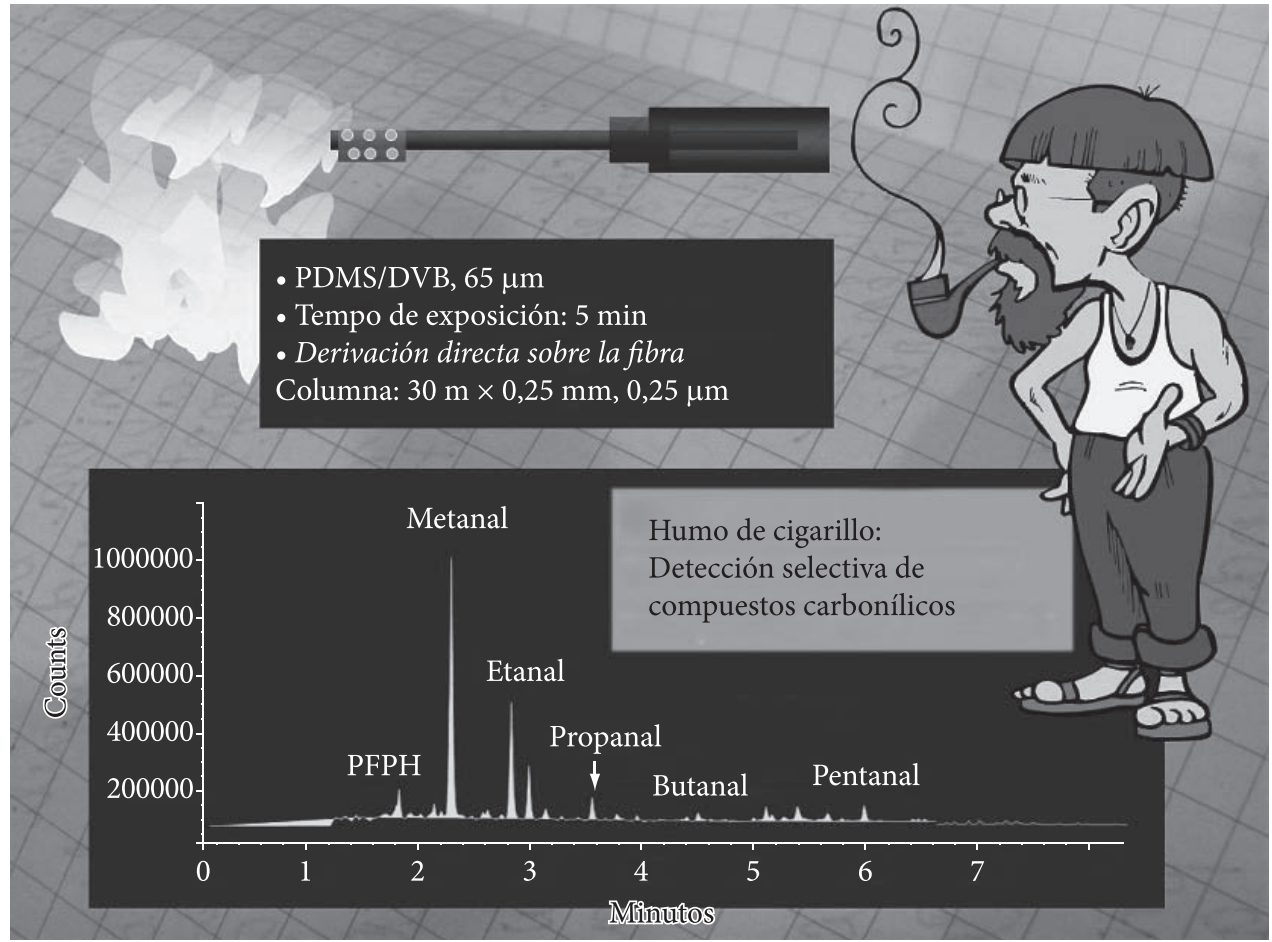

(b)

Figura 17 a) Modo de derivación directa de los analito-target sobre la fibra SPME, previamente saturada con un agente derivatizante (tomado de ref. 67). b) Análisis de sustancias carbonílicas volátiles en el humo de cigarrillo: extracción selectiva por la fibra de SPME saturada con PFPH (pentaflúorfenilhidracina) y detección de hidrazonas formadas in situ por GC-ECD.

de una jeringa, o el interior se rellena con carbón activado $^{73}$. Se denomina HS-SPDE (Headspace Solid-Phase Dynamic Extraction por sus siglas en inglés) a la técnica de muestreo en la que la aguja de una jeringa hermética para muestreo de gases se recubre con polímero absorbente (PDMS), en 
el que se depositan los analitos cuando la jeringa se acciona repetidamente sobre la muestra ${ }^{74}$. El número de ciclos de succión y expulsión del aire determina la cantidad de analitos que se acumulan. Éstos son desorbidos posteriormente en el puerto de inyección del cromatógrafo, tal como se practica con SPME.

Otros ejemplos de técnicas de muestreo derivadas de SPME que usan mayor volumen de fase absorbente son SBSE (Stir Bar Solid-Phase Extraction) y STE (Sorption Tape Extraction). En la primera, la barra magnética de agitación se recubre con polímero absorbente. Luego de su exposición a la solución que contiene los analitos de interés, la barra de agitación se somete a termodesorción para la transferencia de los analitos al cromatógrafo ${ }^{75}$. En STE, una cinta delgada de PDMS se coloca por un tiempo fijo directamente en contacto con la superficie de la planta, la piel o el objeto que se muestrea. Los analitos se transfieren posteriormente al cromatógrafo por termodesorción ${ }^{76}$.

En la microextracción en fase líquida (LPME) los analitos se disuelven en una película de disolvente que se forma en el interior de la aguja de una jeringa, o en su extremo, formando una $\operatorname{gota}^{77}$. El tipo de solvente, y el tamaño o el número de ciclos de uso de la jeringa, son los principales parámetros que se modifican para optimizar la extracción. La revisión hecha por Koning, Janssen y Brinkmann ${ }^{44}$, contiene tablas que presentan referencias representativas del uso de muchas de estas técnicas modernas de preparación de muestras para análisis GC o GC-MS.

\section{Conclusiones}

El éxito del análisis cromatográfico depende fuertemente de una preparación esmerada de la muestra. El analista debe tener en cuenta la naturaleza química de los analitos de interés y de la matriz que los contiene, para seleccionar la técnica de muestreo apropiada. Se han logrado grandes avances en miniaturización, integración, selectividad, eficiencia, reproducibilidad, reducción de costos y robustez de las técnicas de muestreo, extracción y concentración, pero siguen siendo indispensables ciertas prácticas de aseguramiento de la calidad de la cadena analítica, tales como el uso de blancos, sustancias testigo, etapas de limpieza y réplicas.

\section{Agradecimientos}

Los autores agradecen la colaboración en el análisis cienciométrico a Martha Cervantes, Química, M.Sc.

\section{Referencias}

1 Rödel W, Wölm G. Guide to gas chromatography. Heilderberg: Hüthig; 1987.

2 Robards K, Haddad PR, Jackson PE. Principles and practice of modern chromatographic methods. London: Academic Press; 1994.

3 Niessen WM. Current practice of gas chromatographymass spectrometry. New York: Marcel Dekker; 2001. doi: 10.1201/9781420029512.

4 Herbert CG, Johnstone RAW. Mass spectrometry basics. Boca Raton, FL: CRC Press; 2003.

5 Bruner F. Gas chromatographic environmental analysis. New York: VCH; 1993.

6 Shibamoto T. Chromatographic analysis of environmental and food toxicants. New York: Marcel Dekker; 1998.

7 Guillot S, Peytavi L, Bureau S, Boulanger R, Lepoutre JP, Crouzet J et al. Aroma characterization of various apricot varieties using headspacesolid phase microextraction combined with gas chromatography-mass spectrometry and gas chromatography-olfactometry. Food Chemistry 2006; 96(1):147-155. doi: 10.1016/j.foodchem.2005.04.016 
8 Sánchez-Palomo E, Díaz-Maroto MC, PérezCoello MS. Talanta 2005; 66:1152. PMid:18970103. doi:10.1016/j.talanta.2005.01.015

9 Lachenmeier DW, Kroener L, Musshoff F, Madea B. Application of tandem mass spectrometry combined with gas chromatography and headspace solid-phase dynamic extraction for the determination of drugs of abuse in hair samples. Rapid Communications in Mass Spectrometry 2003:17:472. PMid: 12590396. doi:10.1002/rcm.945

10 Thievis M. Mass spectrometry in sports drug testing. New York: John Wiley and Sons; 2010.

11 Cole MD. The analysis of controlled substances. Chichester: Wiley; 2003. doi: 10.1002/0470868007

12 Wong JW, Zhang K, Tech K, Hayward DG, Makovi $\mathrm{CM}$, Krynitsky A et al. Multiresidue pesticide analysis in fresh produce by capillary gas chromatographymass spectrometry/selective ion monitoring (GC-MS/ SIM) and -tandem mass spectrometry (GC-MS/MS). Journal of Agricultural and Food Chemistry 2010; 58(10):5868-5883. PMid: 20199080. doi:10.1021/ jf903854n

13 Pert A, Baron M, Birket J. Review of analytical techniques for arson residues. Journal of Forensic Sciences 2006; 51:1033. PMid: 17018079. doi:10.1111/ j.1556-4029.2006.00229.x

14 Yinon J. Forensic and environmental detection of explosives. Chichester, U.K.: John Wiley and Sons; 1999.

15 Yinon J. Forensic applications of mass spectrometry. Boca Raton: CRC Press; 1995.

16 Montaudo G, Lattimer R. Mass spectrometry of polymers. Boca Raton: CRC Press; 2002.

17 Gordon MH. Principles and applications of gas chromatography in food analysis. Chichester: Ellis Horwood; 1990.

18 Cserháti T, Forgács E. Chromatography in food science and technology. Lancaster, PA: Technomic; 1999.

19 Kim J, Lee S-G, Shin S-C, Kwon Y-D, Park I-K. Male-produced aggregation pheromone blend in platypus koryoensis. Journal of Agricultural and Food Chemistry. 2009; 57:1406. PMid: 19170509. doi: $10.1021 /$ jf8032717

20 Sandra P, Sisalli S, Adao A, Lebel M, Le Fur I. LC-GC Europe. 2006; 19:3311.
21 Rubiolo P, Sgorbini B, Liberto E, Cordero C, Bicchi C. Essential oils and volatiles: sample preparation and analysis. A review. Flavor and Fragrance Journal 2010; 25(5):282-290. doi: 10.1002/fff.1984

22 Malherbe S, Watts V, Nieuwoudt HH, Bauer FF, Du Toit M. Analysis of Volatile Profiles of Fermenting Grape Must by Headspace Solid-Phase Dynamic Extraction Coupled with Gas Chromatography-Mass Spectrometry (HS-SPDE GC-MS): Novel Application To Investigate Problem Fermentations. Journal of Agricultural and Food Chemistry 2009; 57:5161. PMid: 19469561. doi: 10.1021/jf900532v

23 Nikfardjam MP, Maier D. Development of a headspace trap HRGC/MS method for the assessment of the relevance of certain aroma compounds on the sensorial characteristics of commercial apple juice. Food Chemistry 2001; 126:1926. doi: 10.1016/j. foodchem.2010.12.021

24 Flament I. Coffee flavor chemistry. New York: John Wiley and Sons; 2002.

25 Raguso RA, Pellmyr O. Oikos 1998; 81:238. doi: $10.2307 / 3547045$

26 Flamini G, Cioni P, Morelli I. Use of solid-phase micro-extraction as a sampling technique in the determination of volatiles emitted by flowers, isolated flower parts and pollen. Journal of Chromatography A 2003; 998:229. doi:10.1016/S0021-9673(03)00641-1

27 Adlard ER. Chromatography in the petroleum industry. Amsterdam: Elsevier; 1995.

Adams RP. Identification of essential oil components by gas chromatography/mass spectrometry. $4^{\text {th }}$ ed. Carol Stream, IL, USA: Allured Publishing; 2007.

29 Joulain D, König WA. The atlas of spectral data of sesquiterpene hydrocarbons. Hamburg: E. B. Verlag; 1998.

30 Ignat I, Volf I, Popa VI. A critical review of methods for characterisation of polyphenolic compounds in fruits and vegetables. Food Chemistry 2011; 126:1821. doi:10.1016/j.foodchem.2010.12.026

31 Rood D. A practical guide to the care, maintenance, and troubleshooting of capillary gas chromatographic systems. Weinhein: Wiley-VCH; 1999.

32 Chapman JR. Practical organic mass spectrometry: a guide for chemical and biochemical analysis. London: John Wiley and Sons; 1995. 
33 Hübschmann H-J. Handbook of GC/MS. Fundamentals and applications. $2^{\text {nd }}$ ed. Weinheim: Wiley-VCH; 2009.

34 Blau K, Helket J, eds. Handbook of derivatives for chromatography. New York: Wiley; 1993.

35 Toyooka T. Modern derivatization methods for separation science. New York: Wiley; 2002.

36 Moldoveanu SC, David V. Sample preparation in chromatography. Amsterdam: Elsevier; 2002.

37 Pawliszyn J. Sampling and sample preparation for field and laboratory. Amsterdam: Elsevier; 2002.

38 Dean JR. Extraction techniques in analytical sciences. Chichester, U.K.: John Wiley and Sons; 2009. doi:10.1002/9780470682494

39 Dean JR. Extraction methods for environmental analysis. New York: Wiley; 1998.

40 Pawliszyn J. Analytical Chemistry 2003; 75:2593.

41 Smith RM. Journal of Chromatography A 2003; 3:1000.

42 Raynie DE. Analytical Chemistry 2004; 76:4659. PMid:15307773. doi:10.1021/ac040117w

43 Raynie DE. Modern extraction techniques. Analytical Chemistry 2006; 78:3997. PMid:16771537. doi:10.1021/ac060641y

44 Koning S, Janssen H-G, Brinkman UAT. Chromatographia Supplement 2009; 69:S33. doi:10.1365/s10337-008-0937-3

45 Augusto F, Lopes L, Zini C. Trac-Trends in Analytical Chemistry 2003; 22:160. doi: 10.1016/S01659936(03)00304-2

46 Parera J, Santos FJ, Galceran MT. Microwave-assisted extraction versus Soxhlet extraction for the analysis of short-chain chlorinated alkanes in sediments. Journal of Chromatography A 2004; 1046:19.

47 Picó Y, Fernández M, Jose Ruíz M, Font G. Current trends in solid-phase-based extraction techniques for the determination of pesticides in food and environment. Journal of Biochemical and Biophysical Methods 2007; 70:117. PMid:17175029. doi:10.1016/j. jbbm.2006.10.010

48 Díez CC, Traag WA, Zommer P, Marinero P, Atienza J. Comparison of an acetonitrile extraction/partitioning and "dispersive solid-phase extraction" method with classical multi-residue methods for the extraction of herbicide residues in barley samples. Journal of Chromatography A 2006; 1131:11. PMid:16904120. doi:10.1016/j.chroma.2006.07.046
49 Lehotay SJ, Son KA, Kwon H, Koesukwiwat U, Fu W, Mastovska K et al. Comparison of QuEChERS sample preparation methods for the analysis of pesticide residues in fruits and vegetables. Journal of Chromatography A 2010; 1217:2548. PMid:20144460. doi:10.1016/j.chroma.2010.01.044

50 Chaintreau F. Simultaneous distillation-extraction: from birth to maturity-review. Flavor and Fragrance Journal 2001; 16:136. doi:10.1002/ff.967

51 Flotron V, Houessou J, Bosio A, Delteil C, Bermond A, Camel V. Rapid determination of polycyclic aromatic hydrocarbons in sewage sludges using microwaveassisted solvent extraction: Comparison with other extraction methods. Journal of Chromatography A 2003; 999:175. doi: 10.1016/S0021-9673(03)00424-2.

52 Kingston HM, Jassie LB. Introduction to microwave sample preparation. Washington: American Chemical Society; 1988.

53 Kingston HM, Haswell SJ. Microwave-enhanced chemistry. Washington: American Chemical Society; 1997.

54 Rice SL, Mitra S. Microwave-assisted solvent extraction of solid matrices and subsequent detection of pharmaceuticals and personal care products (PPCPs) using gas chromatography-mass spectrometry. Analytica Chimica Acta 2007; 589:125. PMid:17397662. doi:10.1016/j.aca.2007.02.051

55 Fritz JS. Analytical solid-phase extraction. New York: Wiley-VCH; 1999.

56 Thurman EM, Mills MS. Solid-phase extraction. New York: John Wiley and Sons; 1998.

57 Westwood SA. Supercritical fluid extraction and its use in chromatographic sample preparation. Boca Raton, FL: CRC Press; 1993.

58 Wenclawlak B. Analysis with supercritical fluids: extraction and chromatography. Berlin: SpringerVerlag; 1992.

59 Huang BK, Qiu LP, Chu QC, Zhang QY, Gao LH, Zheng HC. Comparison of Headspace SPME with Hydrodistillation and SFE for Analysis of the Volatile Components of the Roots of Valeriana officinalis var. Latifolia. Chromatographia 2009; 69:489. doi:10.1365/ s10337-008-0921-y.

60 Crespo MOP, Yusty MAL. Ecotoxicology and Environmental Safety 2006; 64:400 (2006). 
61 Zuloaga O, Etxebarria N, Fernández LA, Madariaga JM. Comparison of accelerated solvent extraction with microwave-assisted extraction and Soxhlet for the extraction of chlorinated biphenyls in soil samples. Trends in Analytical Chemistry 1998; 17:642. doi: 10.1016/S0165-9936(98)00079-X

62 Gan J, Papiernik SK, Koskinen WC, Yates SR. Evaluation of Accelerated Solvent Extraction (ASE) for Analysis of Pesticide Residues in Soil. Environmental Science and Technology 1999; 33: 3249. doi: 10.1021/ es $990145+$.

63 Zini CA, Zanin KD, Christensen E, Caramão EB, Pawliszyn J. Journal of Agricultural and Food Chemistry 2003; 51:2679. PMid:12696957. doi: 10.1021/ jf026047g.

64 Scheppers SA. Solid phase microextraction. A practical guide. New York: Marcel Dekker; 1999.

65 Balasubramanian S, Panigrahi S. Solid-phase microextraction (SPME) techniques for quality characterization of food products: a Review. Food and Bioprocess Technology 2010; 4:1. doi: 10.1007/ s11947-009-0299-3. PMid:12381104. doi: 10.1007/ s11947-009-0299-3.

66 Wang L, Lord H, Morehead R, Dorman F, Pawliszyn J. Sampling and Monitoring of Biogenic Emissions by Eucalyptus Leaves Using Membrane Extraction with Sorbent Interface (MESI). Journal of Agricultural and Food Chemistry 2002; 50:6281. PMid:12381104. doi:10.1021/jf025577+.

67 Stashenko EE, Martínez JR. Derivatization and solidphase microextraction. Trends in Analytical Chemistry 2004; 23:553. doi:10.1016/j.trac.2004.06.002.

68 Araujo L, Wild J, Villa N, Camargo N, Cubillan D, Prieto A. Determination of anti-inflammatory drugs in water samples, by in situ derivatization, solid phase microextraction and gas chromatography-mass spectrometry. Talanta 2008; 75:111. PMid:18371855. doi:10.1016/j.talanta.2007.10.035.
69 Kolb B, Ettre L. Static headspace-gas chromatography. New York: Wiley-VCH; 1997.

70 Narendra N, Galvão MDS, Madruga MS. Volatile compounds captured through purge and trap technique in caja-umbu (Spondias sp.) fruits during maturation. Food Chemistry 2007; 102:726. doi:10.1016/j.foodchem.2006.06.003

71 Tholl D, Boland W, Hansel A, Loreto F, Rose USR, Schnitzler J-P. Practical approaches to plant volatile analysis. Plant Journal 2006; 45:540. PMid:16441348. doi:10.1111/j.1365-313X.2005.02612.x.

72 Bicchi C, Cordero C, Liberto E, Rubiolo P, Sgorbini B, David F et al. Dual-phase twisters: A new approach to headspace sorptive extraction and stir bar sorptive extraction. Journal of Chromatography A 2005; 1094:9. PMid:16257283. doi:10.1016/j.chroma.2005.07.099.

73 Musshoff F, Lachenmeier DW, Kroener L, Madea B. Automated headspace solid-phase dynamic extraction for the determination of cannabinoids in hair samples. Forensic Science. International 2003; 133:32. doi:10.1016/S0379-0738(03)00047-1.

74 Bagheri H, Babanezhad E, Khalilian F. An interior needle electropolymerized pyrrole-based coating for headspace solid-phase dynamic extraction. Analytica Chimica Acta 2009; 634:209. PMid:19185122. doi:10.1016/j.aca.2008.12.047.

75 Bicchi C, Cordero C, Rubiolo P. A survey on highconcentration-capability headspace sampling techniques in the analysis of flavors and fragrances. Journal of Chromatographic Science 2004; 42:402. PMid:15516279.

76 Sandra P, Bicchi C. Capillary gas chromatography in essential oil analysis. Heilderberg: Hüthig; 1987.

77 Shen G, Lee HK. Headspace liquid-phase microextraction of chlorobenzenes in soil with gas chromatography-electron capture detection. Analytical Chemistry 2003; 75:98. PMid:12530824. doi:10.1021/ac020428b.
Recebido: 03/03/2011

Aceito: 14/03/2011 